\title{
Strain-engineered widely tunable perfect absorption angle in black phosphorus from first principles
}

\author{
Alidoust, Mohammad; Halterman, Klaus; Pan, Douxing; Willatzen, Morten; Akola, Jaakko
}

Published in:

Physical Review B

Link to article, DOI:

10.1103/PhysRevB.102.115307

Publication date:

2020

Document Version

Publisher's PDF, also known as Version of record

Link back to DTU Orbit

Citation (APA):

Alidoust, M., Halterman, K., Pan, D., Willatzen, M., \& Akola, J. (2020). Strain-engineered widely tunable perfect absorption angle in black phosphorus from first principles. Physical Review B, 102(11), [115307].

https://doi.org/10.1103/PhysRevB.102.115307

\section{General rights}

Copyright and moral rights for the publications made accessible in the public portal are retained by the authors and/or other copyright owners and it is a condition of accessing publications that users recognise and abide by the legal requirements associated with these rights.

- Users may download and print one copy of any publication from the public portal for the purpose of private study or research.

- You may not further distribute the material or use it for any profit-making activity or commercial gain

- You may freely distribute the URL identifying the publication in the public portal 


\title{
Strain-engineered widely tunable perfect absorption angle in black phosphorus from first principles
}

\author{
Mohammad Alidoust $\odot,{ }^{1}$ Klaus Halterman $\odot,{ }^{2}$ Douxing Pan, ${ }^{*}$ Morten Willatzen $\odot,{ }^{3, *}$ and Jaakko Akola $\oplus^{1,4}$ \\ ${ }^{1}$ Department of Physics, NTNU Norwegian University of Science and Technology, N-7491 Trondheim, Norway \\ ${ }^{2}$ Michelson Lab, Physics Division, Naval Air Warfare Center, China Lake, California 93555, USA \\ ${ }^{3}$ Department of Photonics Engineering, Technical University of Denmark, Kongens Lyngby DK-2800, Denmark \\ ${ }^{4}$ Computational Physics Laboratory, Tampere University, P.O. Box 692, FI-33014 Tampere, Finland
}

(Received 10 February 2020; revised 1 September 2020; accepted 2 September 2020; published 25 September 2020)

\begin{abstract}
Using the density functional theory of electronic structure, we compute the anisotropic dielectric response of bulk black phosphorus subject to strain. Employing the obtained permittivity tensor, we solve Maxwell's equations and study the electromagnetic response of a layered structure comprising a film of black phosphorus stacked on a metallic substrate. Our results reveal that a small compressive or tensile strain, $\approx 4 \%$, exerted either perpendicular or in the plane to the black phosphorus growth direction, efficiently controls the epsilon-near-zero response and allows perfect absorption tuning from low angle of the incident beam $\theta=0^{\circ}$ to high values $\theta \approx 90^{\circ}$ while switching the energy flow direction. Incorporating the spatially inhomogeneous strain model, we also find that for certain thicknesses of the black phosphorus, near-perfect absorption can be achieved through controlled variations of the in-plane strain. These findings can serve as guidelines for designing largely tunable perfect electromagnetic wave absorber devices.
\end{abstract}

DOI: 10.1103/PhysRevB.102.115307

\section{INTRODUCTION}

Bulk black phosphorus (BP) is an anisotropic semiconductor with two types of chemical bonding. Along two principal crystal directions, the phosphorus atoms form covalent bonds with a puckered honeycomb arrangement, whereas in the third direction atoms interact relatively weakly through van der Waals forces [1-6]. The latter bonding results in a layered configuration consisting of two-dimensional phosphorus sheets. These weakly interacting two-dimensional layers provide a unique opportunity to create different orderings of two-dimensional layers with extremely low-cost and simple operations, including displacement and twist [5-17]. The ordering and number of layers, as well as their deformation, can effectively control the electronic properties of BP-based devices. For instance, the band gap of BP is highly sensitive to the number of BP layers so that a monolayer of BP possesses the largest band gap which decreases by adding more layers. The application of strain into the plane of two-dimensional layers can cause a number of nontrivial phenomena, including manipulation of the band structure, and consequently, electronic response.

Another significant property of BP is its ability to absorb electromagnetic (EM) waves over a broad range of wavelengths, from visible to infrared [8,9,18-36]. Furthermore, the absorption of EM waves by BP can be significantly enhanced by creating layered geometries that generate interference phenomena. These structures can offer advancements in photodetectors and field effect transistors $[33,34,37,38]$.

\footnotetext{
*Present address: Beijing Institute of Nanoenergy and Nanosystems, Chinese Academy of Sciences, No. 30 Xueyuan Road, Haidian District, Beijing 100083, China
}

One of the main challenges in developing modern optical devices is the inherent optical loss that can adversely impact favorable phenomena such as elastic scattering and the transport of optical information. Recently, it was shown that by incorporating detailed electronic band structure effects into the light scattering rate, one will be able to describe the optical loss properties of a material more accurately [39].

Some recent design approaches to control the loss in absorbers involve the use of epsilon-near-zero (ENZ)-based metamaterials [40], where the EM response is described by a permittivity tensor $\overline{\bar{\varepsilon}}$ with at least one component whose real part becomes vanishingly small over certain frequencies. A number of ENZ-based architectures have been fabricated, including subwavelength dielectric coatings with ENZ regions that control the resonant coupling of light [41] and the propagation of a transverse magnetic optical beam through a subwavelength slit. These experiments demonstrated a transmission enhancement when the semiconductor substrate was tuned to its ENZ frequency [42]. Previous ENZ-based absorbers often exploit resonance or interference effects that arise from the large electric-field enhancement and extreme values of the propagation vectors in the ENZ medium. By placing a metal in contact with a material exhibiting ENZ response, the reflected waves from the metal can interfere via coherent perfect absorption [43], whereby the incident EM beam is perfectly coupled to the structure.

In this paper, we address how the application of strain in various directions can be utilized as an efficient experimental knob that controls coherent perfect absorption in a bulk two-dimensional material stacked on a metallic substrate. By performing first-principles calculations, we obtain the anisotropic dielectric response of bulk BP subject to compressive and tensile strain. Incorporating the permittivity tensor from first-principles calculations within Maxwell's equations, 


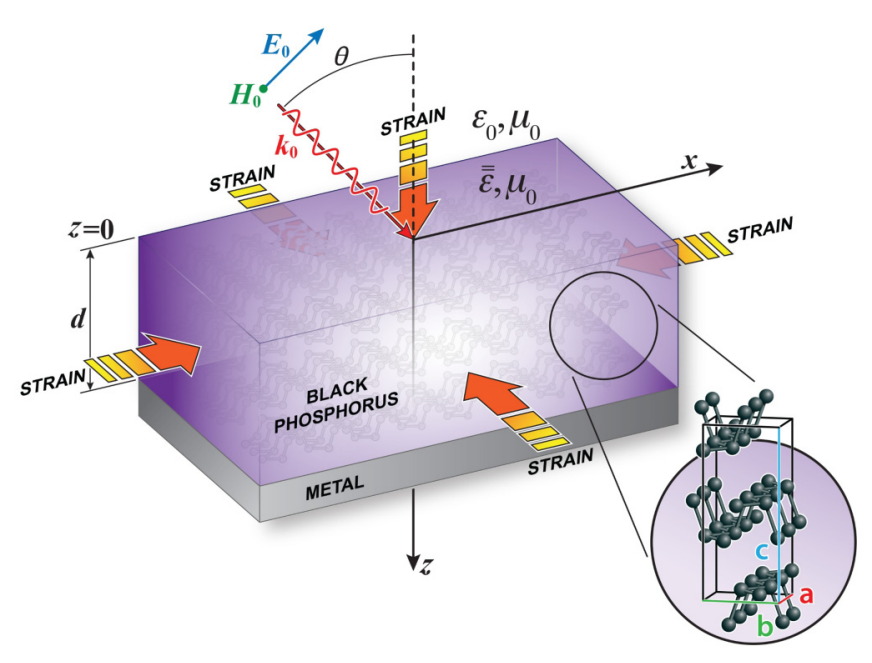

FIG. 1. Schematic configuration involving a slab of bulk black phosphorus of thickness $d$ adjacent to a metallic substrate. The black phosphorus layer is exposed to a transverse magnetic field from the vacuum region where the incident electric field is polarized in the $x-z$ plane, and the magnetic field is polarized along $y$. The incident wave vector $\boldsymbol{k}_{0}$ makes an angle $\theta$ with the $z$ axis. The tensile or compressive strains are applied in the $x-y$ plane and along the $z$ direction (note that the arrows marking applied strain can be reversed corresponding to compressive and tensile strain, respectively). The crystallography principal directions are set as follows: $x \equiv a, y \equiv b$, and $z \equiv c$.

we show that an electromagnetic wave incident on a semiinfinite layered $\mathrm{BP} /$ metallic stack (shown in Fig. 1), can be perfectly absorbed by a strain-controlled ENZ response mechanism. By varying the strain, interference effects can be tuned to achieve perfect absorption over a wide range of incident beam angles $\theta_{P}$, ranging from near-grazing incidence $\left(\theta_{P} \approx\right.$ $\left.0^{\circ}\right)$ to near-normal incidence $\left(\theta_{P} \approx 90^{\circ}\right)$. Also, we show that the application of a low strain value, $\approx 4 \%$, results in energy flow reversal by $180^{\circ}$ within the BP region. Furthermore, we find that when strain is nonuniform along the direction normal to the BP layer, near perfect absorption can arise through controlled variations in the in-plane strain.

The paper is organized as follows. In Sec. II, we summarize the theoretical framework used to describe the proposed EM perfect absorber from atomistic scale. In Sec. III, we present the main findings and characterize how BP-based EM perfect absorber works, including thickness, angle of incident EM beam, and the associated ENZ response. Additional information and discussions are presented in Appendices A and B. Finally, we give concluding remarks in Sec. IV.

\section{METHOD AND APPROACH}

The dielectric function $\tilde{\varepsilon}$ of a system can be defined by the response to an external electric field $\mathbf{E}_{\text {ext }}$

$$
\mathbf{D}(\boldsymbol{r} ; \omega)=\int d \boldsymbol{r} \tilde{\varepsilon}\left(\boldsymbol{r}-\boldsymbol{r}^{\prime} ; \omega\right) \mathbf{E}_{\mathrm{ext}}\left(\boldsymbol{r}^{\prime} ; \omega\right),
$$

where $\boldsymbol{r}$ is location, $\omega$ is the frequency of external field, and $\mathbf{E}$ is the total electric field. If the external field is sufficiently weak (compared with the internal electric fields produced by charge density imbalance and ion interaction), the location and time variation of charge density $\delta n(\boldsymbol{r} ; t)$ can be approximated as a linearly dependent response to the external field

$$
\begin{aligned}
\delta n(\boldsymbol{r} ; t) & =\int d t^{\prime} \int d \boldsymbol{r}^{\prime} \chi\left(\boldsymbol{r}, \boldsymbol{r}^{\prime}, t, t^{\prime}\right) \delta \mathbf{E}_{\mathrm{ext}}\left(\boldsymbol{r}^{\prime}, t^{\prime}\right), \\
\chi\left(\boldsymbol{r}, \boldsymbol{r}^{\prime}, t, t^{\prime}\right) & =\frac{\delta n(\boldsymbol{r} ; t)}{\delta \mathbf{E}_{\mathrm{ext}}\left(\boldsymbol{r}^{\prime}, t^{\prime}\right)} .
\end{aligned}
$$

Within the context of the density functional theory (DFT), a weak variation in the effective potential $V_{\text {eff }}$ of the KohnSham equations results in the variation of charge density

$$
\begin{aligned}
\delta n(\boldsymbol{r} ; t) & =\int d t^{\prime} \int d \boldsymbol{r}^{\prime} \mathcal{K}\left(\boldsymbol{r}, \boldsymbol{r}^{\prime}, t, t^{\prime}\right) \delta \mathrm{V}_{\mathrm{eff}}\left(\boldsymbol{r}^{\prime}, t^{\prime}\right), \\
\mathcal{K}\left(\boldsymbol{r}, \boldsymbol{r}^{\prime}, t, t^{\prime}\right) & =\frac{\delta n(\boldsymbol{r} ; t)}{\delta \mathrm{V}_{\mathrm{eff}}\left(\boldsymbol{r}^{\prime}, t^{\prime}\right)} .
\end{aligned}
$$

By equating Eqs. (2) and (3), one can evaluate the dielectric response function $\chi$ for an interacting electron system using the Kohn-Sham response function $\mathcal{K}$ from atomisticscale DFT simulations [44]. A well-known approximation that highly simplifies the calculations is the random phase approximation (RPA), neglecting the exchange-correlation contribution once the ground-state electron density has been computed [45].

In this work, the atomistic-scale calculations of the dielectric response were performed in the framework of DFT using the GPAW program package, which utilizes the projectoraugmented wave (PAW) method for the interaction between valence electrons and ion cores $[39,46,47]$. The gradientcorrected functional by Perdew-Burke-Ernzerhof (PBE) was employed for the exchange-correlation energy (electronic band structure and density of states). We have used a relatively high value, i.e., $6.0 \mathbf{k}$ points per $\AA^{-1}$ for the $\mathbf{k}$-point density in order to grid $\mathbf{k}$ space on the basis of the Monkhorst-Pack scheme. The plane-wave cutoff for the kinetic energy was set to $800 \mathrm{eV}$ and 60 unoccupied electronic bands were chosen for the unit cell of eight atoms with a convergence on the first 50 bands to avoid any artificial effects that strain may induce around the Fermi energy. Correspondingly, the width of the Fermi-Dirac distribution was set to $0.01 \mathrm{eV}$. RPA is used for the density response function, and the integrations to obtain the dielectric response are performed by the linear tetrahedron interpolation scheme as implemented in GPAW [39]. A small imaginary part was added to the frequencies with $\delta \omega=0.01 \mathrm{eV}$.

To simulate BP under strain, we introduce the strain parameters $\epsilon_{i i}$, (for $i=x, y, z$ ) corresponding to the normalized percentage of uniform shrinkage with respect to relaxed unit cell. We define, $a=\epsilon_{x x} a_{0}, b=\epsilon_{y y} b_{0}$, and $c=\epsilon_{z z} c_{0}$, where $a$, $b$, and $c$ are the three strained unit cell axis lengths, and the unstrained unit cell axis lengths are $a_{0}, b_{0}$, and $c_{0}$. The exact values of these parameters and the location of phosphorus atoms in the unstrained unit cell are summarized in Table II of Appendix B. We also present the electronic band structure and density of states for the unstrained BP as well as its strained forms in Appendix B. Hence, in this notation, $\epsilon_{x x}=\epsilon_{y y}=$ $\epsilon_{z z}=1.0$ corresponds to zero strain, and, e.g., a compressive or tensile strain of $\mp 10 \%$ in the $z$ direction is denoted by $\epsilon_{z z}=0.9$ and $\epsilon_{z z}=1.1$, respectively. Note that both unit cell 
TABLE I. Dielectric response and strain parameters for bulk BP calculated by DFT. The strains $\left(\epsilon_{\|}, \epsilon_{z z}\right)$ and permittivity components $\left(\varepsilon_{1 x}, \varepsilon_{1 z}\right)$ are presented for the given frequency $(\omega)$.

\begin{tabular}{ccccc}
\hline \hline$\omega(\mathrm{eV})$ & $\epsilon_{\|}$ & $\epsilon_{z z}$ & $\varepsilon_{1 z}$ & $\varepsilon_{1 x}$ \\
\hline 0.573 & 1.00 & 0.90 & $0.000996+0.374 i$ & $7.577+0.152 i$ \\
0.380 & 1.00 & 0.94 & $0.000919+0.0527 i$ & $9.419+0.0371 i$ \\
0.124 & 1.00 & 1.00 & $0.00153+0.0176 i$ & $11.294+0.00779 i$ \\
22.64 & 0.90 & 1.00 & $0.000149+0.196 i$ & $0.160+0.140 i$ \\
22.64 & 1.00 & 1.00 & $0.233+0.142 i$ & $0.198+0.0979 i$ \\
22.64 & 1.10 & 1.00 & $0.341+0.0688 i$ & $0.347+0.0402 i$ \\
\hline \hline
\end{tabular}

parameters $a, b, c$ and correspondingly, the location of atoms are renormalized in the presence of strain. The permittivity tensor $\overline{\bar{\varepsilon}}$ takes the following biaxial form that is valid for BP belonging to the $D_{2 h}$ point group:

$$
\overline{\bar{\varepsilon}}_{n}=\varepsilon_{n x} \hat{\boldsymbol{x}} \hat{\boldsymbol{x}}+\varepsilon_{n y} \hat{\boldsymbol{y}} \hat{\boldsymbol{y}}+\varepsilon_{n z} \hat{z} \hat{z},
$$

where $n$ denotes either the vacuum region $(n=0)$ or $\mathrm{BP}$ region $(n=1)$. In general, from symmetry considerations, the permeability tensor $\overline{\bar{\mu}}$ is also biaxial; however for nonmagnetic BP, $\overline{\bar{\mu}}=\mu_{0} \overline{\bar{I}}$. Note that we make use of symbols " $\epsilon_{x x, y y, z z}$ " for the strain whereas " $\varepsilon_{n x, n y, n z}$ " are used for denoting the dielectric response.

We next demonstrate how the BP structure in the lowpermittivity regime can exhibit perfect absorption of EM waves over a broad range of incident angles and system parameters, thus revealing a practical platform for the control of EM radiation. We investigate the reflection and absorption of EM waves from the layered configuration shown in Fig. 1, which comprises a planar BP material adjacent to a metallic substrate with perfect conductivity (PEC). The electric field of the incident wave is polarized in the $x-z$ plane, so that only the permittivity components $\varepsilon_{1 x}$ and $\varepsilon_{1 z}$ participate in the overall EM response. The plane wave is incident from the vacuum region with wave vector $\boldsymbol{k}_{0}$ in the $x-z$ plane: $\boldsymbol{k}_{0}=\hat{\boldsymbol{x}} k_{0 x}+\hat{z} k_{0 z}$. Since there are no off-diagonal components, the TM (transverse magnetic) and TE (transverse electric) modes are decoupled.

TABLE II. Lattice parameters $\left(a_{0}, b_{0}, c_{0}, \alpha, \beta, \gamma\right)$ and the normalized location of phosphorus atoms, $\mathrm{P}_{i}, i=1,2, \ldots, 8,(x, y, z)$ for the relaxed bulk black phosphorus unit cell at zero strain.

\begin{tabular}{cccc}
\hline \hline$a_{0}(\AA)$ & $b_{0}(\AA)$ & $c_{0}(\AA)$ & $\operatorname{Vol}\left(\AA^{3}\right)$ \\
\hline 3.31590 & 4.50640 & 10.44520 & 156.080247 \\
$\alpha(\mathrm{deg})$ & $\beta(\mathrm{deg})$ & $\gamma(\mathrm{deg})$ & \\
$90^{\circ}$ & $90^{\circ}$ & $90^{\circ}$ & \\
Atom & $x(\AA)$ & $y(\AA)$ & $z(\AA)$ \\
$\mathrm{P}_{1}$ & 0.25000 & 0.08486 & 0.07454 \\
$\mathrm{P}_{2}$ & 0.75000 & 0.91514 & 0.37093 \\
$\mathrm{P}_{3}$ & 0.25000 & 0.58486 & 0.37093 \\
$\mathrm{P}_{4}$ & 0.75000 & 0.41514 & 0.07454 \\
$\mathrm{P}_{5}$ & 0.75000 & 0.08486 & 0.57454 \\
$\mathrm{P}_{6}$ & 0.25000 & 0.91514 & 0.87093 \\
$\mathrm{P}_{7}$ & 0.75000 & 0.58486 & 0.87093 \\
$\mathrm{P}_{8}$ & 0.25000 & 0.41514 & 0.57454 \\
\hline \hline
\end{tabular}

The incident electric and magnetic fields thus have the following forms

$$
\begin{aligned}
\boldsymbol{E} & =\left(E_{x 0} \hat{\boldsymbol{x}}+E_{z 0} \hat{z}\right) e^{i\left(k_{0 x} x+k_{0 z} z-\omega t\right)}, \\
\boldsymbol{H} & =H_{y 0} \hat{\mathbf{y}} e^{i\left(k_{0 x} x+k_{0 z} z-\omega t\right)} .
\end{aligned}
$$

Due to continuity in the transverse electric field, $k_{0 x}$ is invariant across the interface with $k_{0 x}=k_{0} \sin \theta, k_{0 z}=k_{0} \cos \theta$, and $k_{0}=\omega / c$. For both the vacuum and BP regions, we implement Maxwell's equations for time harmonic fields $\left(e^{-i \omega t}\right)$,

$$
\begin{aligned}
\nabla \times \boldsymbol{E}_{j} & =i \omega \mu_{0} \boldsymbol{H}_{j}, \\
\nabla \times \boldsymbol{H}_{j} & =-i \omega \boldsymbol{D}_{j},
\end{aligned}
$$

where $j=0$ or 1 to identify either the vacuum or BP regions, respectively. When expressing the EM fields in BP as plane waves, the propagation vector there, $\boldsymbol{k}_{1}$, replaces the spatial derivatives, transforming Maxwell's equations into the forms, $\boldsymbol{k}_{1} \times \boldsymbol{E}_{1}=\omega \mu_{0} \boldsymbol{H}_{1}$ and $\boldsymbol{k}_{1} \times \boldsymbol{H}_{1}=-\omega \overline{\bar{\varepsilon}}_{1} \varepsilon_{0} \boldsymbol{E}_{1}$. These two equations together result in the following expression for the $\boldsymbol{E}_{1}$ field in $\boldsymbol{k}$ space: $\boldsymbol{k}_{1} \times\left(\boldsymbol{k}_{1} \times \boldsymbol{E}_{1}\right)=-k_{0}^{2} \overline{\bar{\varepsilon}}_{1} \boldsymbol{E}_{1}$. Upon using the identity $\boldsymbol{k}_{1} \times\left(\boldsymbol{k}_{1} \times \boldsymbol{E}_{1}\right)=\boldsymbol{k}_{1}\left(\boldsymbol{k}_{1} \cdot \boldsymbol{E}_{1}\right)-\boldsymbol{k}_{1}^{2} \boldsymbol{E}_{1}$, one finds the dispersion equation for the BP region:

$$
\left(k_{0 x}^{2}-\varepsilon_{1 y} k_{0}^{2}+k_{1 z}^{2}\right)\left(\varepsilon_{1 x} \varepsilon_{1 z} k_{0}^{2}-\varepsilon_{1 x} k_{0 x}^{2}-\varepsilon_{1 z} k_{1 z}^{2}\right)=0 .
$$

Solving for the roots in Eq. (7) results in two types of solutions for $k_{1 z}$. If there is a $y$ component to the electric field, then we have TE modes with $k_{1 z}= \pm \sqrt{\varepsilon_{1 y} k_{0}^{2}-k_{0 x}^{2}}$. For the case of interest, the electric field is polarized in the $x$ and $z$ directions (TM modes) with the following wave vectors for each region:

$$
k_{1 z}= \pm \sqrt{\varepsilon_{1 x}\left(k_{0}^{2}-\frac{k_{0 x}^{2}}{\varepsilon_{1 z}}\right)}, \quad k_{0 z}= \pm \sqrt{k_{0}^{2}-k_{0 x}^{2}} .
$$

Thus it is clear that due to the initial TM polarization state, only $\varepsilon_{1 x}$ and $\varepsilon_{1 z}$ contribute to the EM response of BP.

For the configuration shown in Fig. 1, where the $x-y$ plane is translationally invariant and the thickness along the $z$ axis is finite, the magnetic field component in the vacuum region, $\boldsymbol{H}_{0}$, is written in terms of incident and reflected waves: $H_{y 0}=\left(e^{i k_{0 z} z}+r e^{-i k_{0 z} z}\right) e^{i k_{0 x} x}$, where $r$ is the reflection coefficient. From the magnetic field component, we can use Eqs. (6) to easily deduce the electric field components. For BP region, the general form of the $\boldsymbol{E}$ field is a linear combination of waves with wave vectors given in Eq. (8): $E_{x 1}=\left(a_{1} e^{i k_{1 z} z}+\right.$ $\left.a_{2} e^{-i k_{1 z} z}\right) e^{i k_{0 x} x}$. To construct the remaining $\boldsymbol{E}$ and $\boldsymbol{H}$ fields we use Maxwell's equations to get the following relations

$$
\begin{aligned}
& \partial_{z} H_{y 1}=i \omega \varepsilon_{0} \varepsilon_{1 x} E_{x 1}, \\
& k_{0 x} H_{y 1}=-\omega \varepsilon_{0} \varepsilon_{1 z} E_{z 1}, \\
& \partial_{z} E_{x 1}-i k_{0 x} E_{z 1}=i \omega \mu_{0} H_{y 1} .
\end{aligned}
$$

Upon matching the tangential electric and magnetic fields at the vacuum/BP interface, and using the boundary conditions of vanishing tangential electric fields at the metallic ground plane, it is straightforward to determine the unknown coefficients $a_{1}, a_{2}$, and $r$.

We assume that the metallic substrate is perfectly conducting. For example, silver at a frequency of $20 \mathrm{eV}$ and corresponding skin depth $\sim 1 \times 10^{-3} \mu \mathrm{m}$ behaves as a nearly 
perfect reflector [43]. At high frequencies, the metallic substrate may no longer serve as an effective reflector, which in turn diminishes the processes involved in coherent perfect absorption. Thus, the device should be designed to operate below the plasma frequency of the chosen metal. The reflection coefficient $r$ is found to be

$$
r=\frac{2 \varepsilon_{1 z} k_{0 z} k_{1 z} \cos \left(k_{1 z} d\right)}{\varepsilon_{1 z} k_{0 z} k_{1 z} \cos \left(k_{1 z} d\right)-i\left(\varepsilon_{1 z} k_{0}^{2}-k_{0 x}^{2}\right) \sin \left(k_{1 z} d\right)}-1 .
$$

The coefficients $a_{1}$ and $a_{2}$ are simply related: $a_{1} / a_{2}=$ $-e^{-2 i k_{1 z} d}$, where

$$
a_{1}=\frac{\eta_{0} k_{0 z}}{k_{0} \cos \left(k_{1 z} d\right)} \frac{e^{-i k_{1 z} d}}{\frac{\varepsilon_{1 z} k_{02} k_{1 z}}{\varepsilon_{1 z} k_{0}-k_{0 x}^{2}}-i \tan \left(k_{1 z} d\right)}
$$

and $\eta_{0}=\sqrt{\mu_{0} / \varepsilon_{0}}$ is the impedance of free space. The reflection coefficient has the property that $r\left(k_{0 z}\right)=r^{-1}\left(-k_{0 z}\right)$, which simplifies the solution process later since finding a complex pole (related to $-k_{0 z}$ ), is equivalent to finding the perfect absorption modes for $k_{0 z}[48,49]$.

In determining the absorptance $A$ of the black phosphorus system, we consider energy conservation and implement the time-averaged Poynting vector $\boldsymbol{S}$, given by $\boldsymbol{S}=\frac{1}{2} \Re\left\{\boldsymbol{E} \times \boldsymbol{H}^{*}\right\}$. Considering the component in the direction perpendicular to the interfaces (the $z$ direction), and inserting the electric and magnetic fields calculated for the vacuum region, we find

$$
A=1-|r|^{2} .
$$

Here $A$ is defined as $S_{z} / S_{0}$, in which $S_{0} \equiv k_{0 z} /\left(2 \varepsilon_{0} \omega\right)$ is the time-averaged Poynting vector for a plane wave traveling in the $z$ direction. When discussing the direction of energy flow, it is insightful to consider the angle $\theta_{S}$ that the Poynting vector makes at $\mathrm{BP} /$ vacuum interface. We thus consider $\tan \theta_{S}=$ $\Re\left(S_{x}\right) / \Re\left(S_{z}\right)$. Inserting the calculated electric and magnetic fields, we find the following general relationships: For the direction of energy flow in the vacuum region, we have,

$$
\tan \theta=\frac{1-|r|^{2}}{|1+r|^{2}} \tan \theta_{S} .
$$

Here $r$ is a function of the permittivities $\varepsilon_{1 x}$ and $\varepsilon_{1 z}$, incident angle $\theta$, frequency $\omega$, and thickness $d$ [see Eq. (10)]. Consequently, if there is no reflected wave $(r=0)$, the angle of perfect absorption $\theta_{P}$ equals the direction of energy flow. This is not necessarily the case just inside BP, where the angle of the energy flow inside $\mathrm{BP}$ at $\mathrm{BP} /$ vacuum interface obeys the simple relation:

$$
\tan \theta_{S}=\Re\left\{\frac{1}{\varepsilon_{1 z}}\right\} \tan \theta_{P} .
$$

Having established the methods for determining the absorption and reflection coefficients, we now consider a range of material and geometrical parameters that leads to perfect absorption in the low-permittivity regime. To clarify the coupling of the incident beam to fast wave modes, it is important to examine the corresponding waveguide modes of the structure. The poles of the reflection coefficient, where the denominator in Eq. (10) vanishes, yield the allowed modes:

$$
\tan \left(k_{1 z} d\right)+\frac{i \varepsilon_{1 z} k_{0 z} k_{1 z}}{\varepsilon_{1 z} k_{0}^{2}-k_{0 x}^{2}}=0 .
$$

The transcendental equation [Eq. (15)] provides four types of solutions for the propagation constant $k_{0 x}$ due to the \pm signs for $k_{1 z}$ and $k_{0 z}$ [50]. The branch leading to perfect absorption corresponds to both $k_{1 z}<0$ and $k_{0 z}<0$, yielding fast-wave $\left(k_{0 x} / k_{0}<1\right)$, nonradiative $\left(k_{0 x} \in \mathfrak{R}\right)$ modes that represent a coherent superposition of waves that propagate without loss along the BP surface [43]. Once the propagation constants are found, they can be correlated with the parameters that lead to the angles of perfect absorption, $\theta_{P}$, via $\theta_{P}=\arcsin \left(k_{0 x} / k_{0}\right)$. As an alternative approach for finding perfect absorption, we also match the effective field impedance of the incident plane wave in free space, $\mathcal{Z}_{0}$, to that of BP structure $\mathcal{Z}_{1}$, where we define $\mathcal{Z}_{i}=E_{x i} /\left.H_{y i}\right|_{z=0}$. These solutions can then be compared with the waveguide modes found in Eq. (15).

\section{RESULTS AND DISCUSSIONS}

In what follows, we study two scenarios for a strained device. In the first case, we assume that an externally applied strain is distributed uniformly throughout the system. In the second case, a linearly distributed strain model is implemented to address an example of nonuniformly strained devices.

\section{A. Uniformly strained system}

We have computed the permittivity tensor $\overline{\bar{\varepsilon}}_{1}(\omega)$ of bulk BP by the DFT method. Since BP belongs to the orthorhombic point group $\mathrm{D}_{2 \mathrm{~h}}$, there should be, in principle, differences in the EM response when strain is applied along either the $x$ or $y$ directions. For simplicity, we consider here situations where the in-plane strain is applied equally in the $x$ and $y$ directions, so that $\epsilon_{x x}=\epsilon_{y y}=\epsilon_{\|}$. The strain is varied in increments of $2 \%$, ranging from $-10 \%$ to $+10 \%$ for both the in-plane $\epsilon_{\|}$ and perpendicular strains $\epsilon_{z z}$. Within a simple Drude model formalism, ENZ responses occur in small regions around the plasma frequency, and for bulk BP, there are several frequencies around which the permittivity is zero. Thus, depending on the strain values, multiple ENZ modes can be accessible over a wide range of frequencies. This adds to the fact that the diagonal components of $\overline{\bar{\varepsilon}}_{1}(\omega)$ in general have real parts that vanish at different frequencies. For coherent perfect absorption to take place, all nonzero components of $\overline{\bar{\varepsilon}}_{1}(\omega)$ take part in the EM response, however $\varepsilon_{1 z}$ plays the greatest role due in part to the finite-size effects (in the $z$ direction) and the corresponding $z$ component of the electric field contributing to the necessary interference effects responsible for perfect absorption.

To illustrate the relationship between the ENZ response and strain, we show in Fig. 2(a), the ENZ frequency $\omega_{p}$ where $\operatorname{Re}\left\{\varepsilon_{1 z}\left(\omega_{p}\right)\right\}=0$, plotted as a function of strain in the $z$ direction. The corresponding imaginary part of the dielectric response $\varepsilon_{1 z}(\omega)$ is shown in Fig. 2(b). Here, we have considered a low frequency regime, i.e., $0.1 \mathrm{eV} \lesssim \omega \lesssim 0.6 \mathrm{eV}$, although ENZ scenarios arise for lower frequencies as well. From Fig. 2(a), it is seen that $\omega_{p}$ declines nearly linearly as 

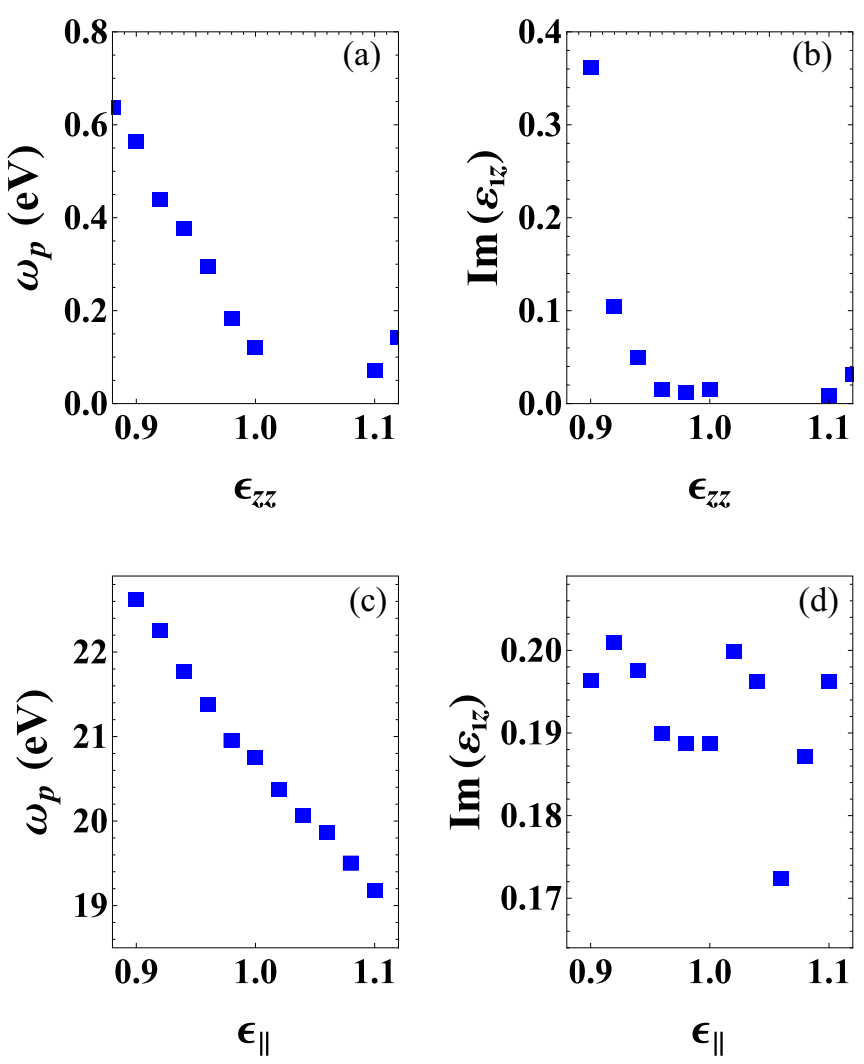

FIG. 2. Top row: (a),(b) Computed frequencies $\omega_{p}$ from DFT that lead to an ENZ response in bulk black phosphorus as a function of strain $\epsilon_{z z}$. The system is unstrained in the plane of the sample $\left(\epsilon_{\|}=\right.$ 1.0). Bottom row: (c),(d) The same as a function of strain $\epsilon_{\|}$where the system is unstrained in the direction normal to the plane of the sample $\left(\epsilon_{z z}=1.0\right)$.

the compressive strain relaxes from $\epsilon_{z z}=0.9$, until reaching the unstrained state at $\epsilon_{z z}=1$. The imaginary component in Fig. 2(b) also declines but much more rapidly. Indeed, the application of a compressive strain less than $6 \%$ results in a vanishingly small imaginary part of $\varepsilon_{1 z}(\omega)$ for these ENZ modes, which is a favorable situation for coherent perfect absorption [43]. When optimizing the BP absorber and limiting undesirable Joule heating, we focus on low to moderate dissipation, so that only data with $\operatorname{Im}\left(\varepsilon_{1 z}\right) \leqslant 0.4$ are shown.

In Figs. 2(c) and 2(d), the system is now unstrained in the $z$ direction, and there are compressive and tensile strains within BP plane. A higher frequency regime is also considered where $19 \mathrm{eV} \lesssim \omega \lesssim 23 \mathrm{eV}$. Moving from a compressive to tensile strain [Fig. 2(c)] causes the ENZ frequency to decline nearly linearly over the whole range of strains. The imaginary component of the dielectric response $\varepsilon_{1 z}$ [Fig. 2(d)] reflects slight changes in the dissipative nature of the system as the in-plane strain varies. However, the effect is weaker at these higher frequencies compared to what was observed in Fig. 2(b). Nonetheless each scenario considered here reveals that strain has a considerable impact on the electromagnetic response of our BP absorber. It is worth mentioning that an in-plane tensile strain of $10 \%$ can open a gap in the band structure of bulk BP as shown in Fig. 10(c) of Appendix B. The band structure plots demonstrate a direct link between
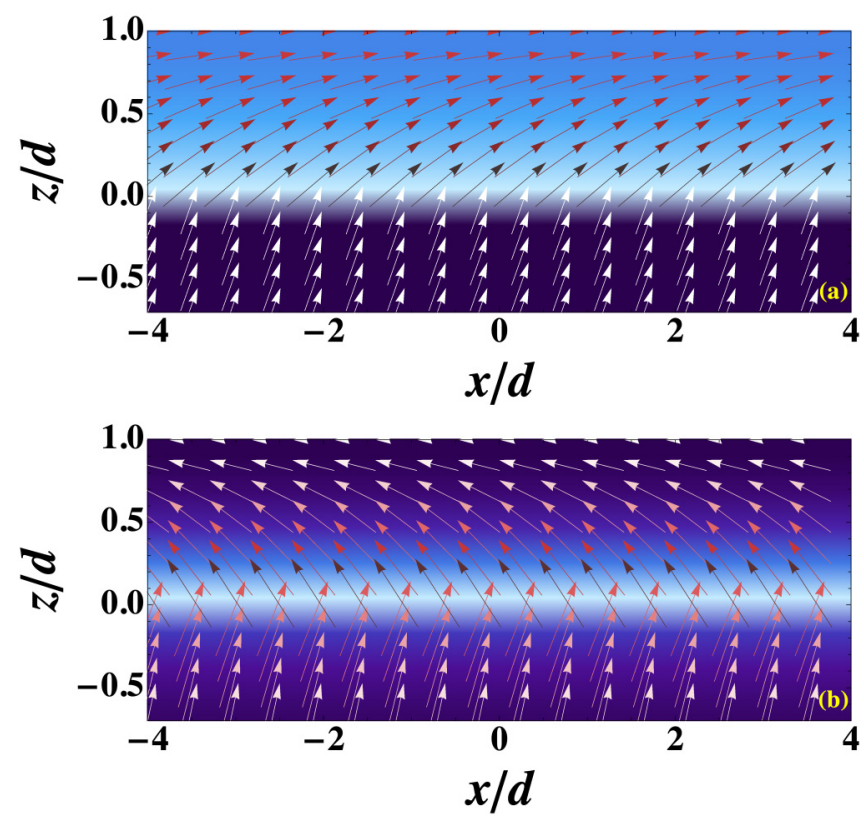

FIG. 3. Directional control of energy flow through strain: Twodimensional maps illustrating the behavior of the time-averaged Poynting vector for a plane wave incident on a black phosphorus film with metallic substrate. The incident angle corresponds to $\theta=34.1^{\circ}$, and the frequency is set at $20.7 \mathrm{eV}$. In (a) the strain parameters correspond to $\epsilon_{\|}=1.04$, leading to perfect absorption, while for (b) the system is unstrained $\left(\epsilon_{\|}=1\right)$. For both cases there is no strain in the $z$ direction $\left(\epsilon_{z z}=1\right)$. The film thickness is $d=5.42 \times 10^{-3} \mu \mathrm{m}$. The interface separating the black phosphorus from the vacuum region is located at $z=0$ and the vacuum region corresponds to $z<0$.

the band crossings (and gap opening) feature of strain to the dissipation part of the dielectric response. As the number of band crossings increases, the probability rate for interband transitions grows, and consequently, BP hosts higher loss rates (see Appendix B).

To depict how strain can influence energy flow in $\mathrm{BP} /$ metallic system, we plot the spatial profile of the timeaveraged Poynting vector in Fig. 3. The arrows indicate the direction of energy flow. The BP layer has a thickness $d=$ $5.42 \times 10^{-3} \mu \mathrm{m}$, which has been normalized between $0<$ $z / d<1$, while the vacuum region occupies the space $z / d<0$ (see Fig. 1). In Fig. 3(a), a 4\% in-plane tensile strain is applied to the BP plane where the incident wave makes a representative angle of $\theta=34.1^{\circ}$ at the frequency of $\omega=20.7 \mathrm{eV}$. The chosen angle corresponds to the perfect absorption angle $\theta_{P}$, and thus all incident EM energy goes into BP layer. For the given value of the tensile strain, the permittivity tensor of BP has components, $\varepsilon_{1 z} \approx 0.092+0.145 i$, and $\varepsilon_{1 x} \approx 0.105+$ $0.192 i$. From Eq. (14), we find that the incident EM wave undergoes significant refraction immediately after entering BP with the wave energy directed at $\theta_{S} \approx 64^{\circ}$. Further inside BP medium, the Poynting vector of the incident EM wave bends increasingly until the energy flows nearly parallel $\left(\theta_{S} \rightarrow 90^{\circ}\right)$ close to the surface of the metal $(z / d \approx 1)$. In Fig. $3(\mathrm{~b})$ the strain is switched off, producing a dielectric response with $\varepsilon_{1 z} \approx-0.085+0.230 i$, and $\varepsilon_{1 x} \approx 0.073+0.265 i$, and the rest of the parameters remain intact. The exertion of only $4 \%$ 

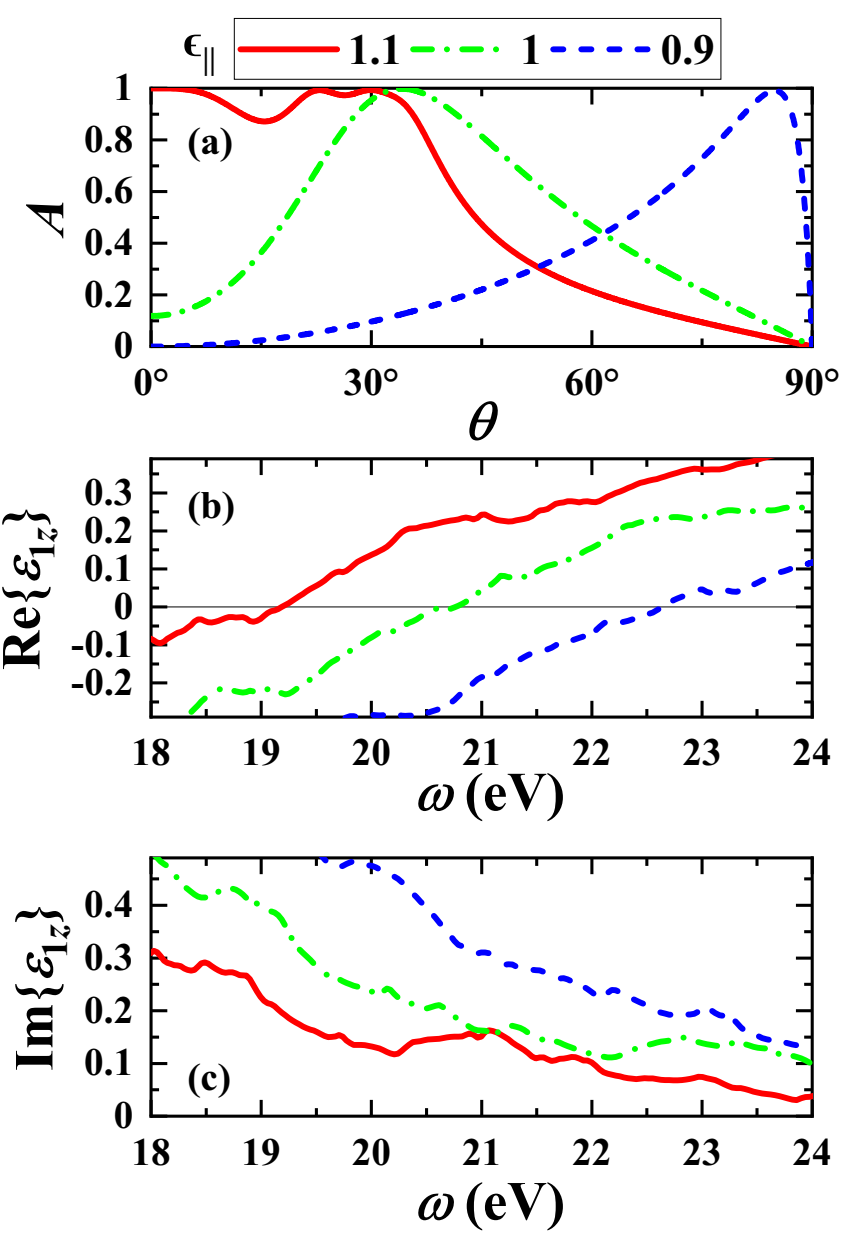

FIG. 4. (a) Absorptance as a function of the incident angle $\theta$ for three in-plane strains $\epsilon_{\|}$, as shown in the legend. The frequency is set at $\omega=22.6 \mathrm{eV}$. (b),(c) The corresponding real and imaginary parts of the permittivity component $\varepsilon_{1 z}$ (obtained by DFT) are shown as a function of the incident wave frequency.

of in-plane tensile strain changes the energy flow direction by nearly $180^{\circ}$. This follows from the strain-induced sign change of the real part of $\varepsilon_{1 z}$. Near the interface in the vacuum, we find from Eq. (13) that the direction of the net flow of energy is shifted slightly to $\theta_{S} \approx 38.5^{\circ}$ as BP now partially reflects some of the energy of the incident wave.

The BP perfect absorber in Fig. 1 can be tailored to absorb EM energy over a wide range of incident angles. To illustrate this, Fig. 4(a) shows the absorptance of the incoming plane wave as a function of its incident angle $\theta$. Each curve represents a different in-plane strain value of the two-dimensional BP sheet (see legend). The strains $\epsilon_{\|}=0.9,1.0$, and $\epsilon_{\|}=$ 1.1 correspond to BP film thicknesses $d=1.5 \times 10^{-4} \mu \mathrm{m}$, $d=0.012 \mu \mathrm{m}$, and $d=0.18 \mu \mathrm{m}$, respectively. We will show below that the thickness $d$ and angle $\theta$ have an intricate relationship that must be satisfied to achieve perfect absorption.

To understand the angular dependence of the absorption, it is important to have an accurate characterization of the EM response in BP, in particular $\varepsilon_{1 z}$. For perfect absorbers in the ENZ or low permittivity regimes, the imaginary part of the permittivity plays a crucial role in determining which incident angles are the perfect absorption angles. Indeed, while optimizing system parameters for practical devices, it is important to keep in mind that when there is a low dissipation in the medium, a very thin layer is required to achieve perfect absorption. As the dissipation increases (e.g., due to changing $\omega$ or $\epsilon_{z z}$ and $\left.\epsilon_{\|}\right)$thicker BP layers are needed. Thus, it is imperative to clarify the frequency dispersive nature of permittivity. In Figs. 4(b) and 4(c), the real and imaginary parts of $\varepsilon_{1 z}$ are shown as a function of frequency. When no strain is exerted [Fig. 4(a)], the maximum absorption occurs in the range $35^{\circ} \lesssim \theta \lesssim 45^{\circ}$. A tensile strain of $10 \%$ then widens the range of perfect absorption angles to fall within $0^{\circ} \lesssim \theta \lesssim 40^{\circ}$. Upon changing the type of strain to compressive, near perfect absorption arises for incident waves at near grazing, with $\theta \approx 85^{\circ}$. These results demonstrate that the application of a low strain along the principal crystallographic directions of BP structure can effectively control the perfect absorption of the incident beam. This occurs for a specific incident angle that correlates with the proper ENZ modes (see Fig. 2).

To further shed light on this phenomenon, in Fig. 5 we plot the absorptance as a function of the incident beam angle and BP thickness. The strain values are labeled in each figure, and the top row of Fig. 5 displays the strain applied orthogonal to the planar interface (along the $z$ direction), whereas in the bottom row in-plane strain is applied (in the $x-y$ plane). The relevant components of the permittivity tensor for the considered strains and frequencies are listed in Table I. For our geometry and polarization state of the incident wave, the component $\varepsilon_{1 x}$ plays a limited role in the absorption of EM energy, but it is listed for completeness.

For the top row in Fig. 5, we consider the compressive strains (a) $\epsilon_{z z}=0.9$ and (b) $\epsilon_{z z}=0.94$, while (c) shows the unstrained case with $\epsilon_{z z}=1$. The frequencies have been chosen to correlate with $\omega_{p}$ in Fig. 2(a), so that an ENZ response is generated for each strain value. Thus, we have used the values (a) $\omega=0.57$, (b) $\omega=0.38$, and (c) $\omega=0.12 \mathrm{eV}$, with each generating different levels of dissipation according to Fig. 2(b) (see also Table I). Since the dissipative response declines as the strain parameter changes from an unstrained state, $\epsilon_{z z}=1.0$, to compressive at $\epsilon_{z z}=0.9$, we have a controllable platform in which EM absorption is dependent on both frequency and strain.

In Figs. 5(d) and 5(f), the frequency of the incident wave is now fixed at $\omega=22.6 \mathrm{eV}$ while the system goes from compressive strain with $\epsilon_{\|}=0.9$ to tensile strain with $\epsilon_{\|}=1.1$. For the tensile strain case, there is a small real part in the permittivity component $\varepsilon_{1 z}$, which vanishes as the system undergoes compressive strain. This ENZ state generates a moderate amount of dissipation which weakens as the inplane strain parameter $\epsilon_{\|}$increases.

The results in Fig. 5 reveal that both compressive and tensile strain can generate perfect absorption over a wide range of $\theta$ and thickness values. In particular, Figs. 5(a)-5(c) show that as $\epsilon_{z z}$ increases, there exists a broader range of incident angles that result in perfect absorption. This follows from a reduction in the dissipative response of $\varepsilon_{1 z}$ as the compressive strain is reduced (see Fig. 2). This behavior is consistent with Weyl semimetal absorbers that have tunable dissipation [51-58] and anisotropic ENZ coherent perfect absorbers [43]. Overall, for an incoming wave at near grazing incidence $\left(\theta \sim 90^{\circ}\right)$ to be fully absorbed, very thin subwavelength BP layers are needed, 

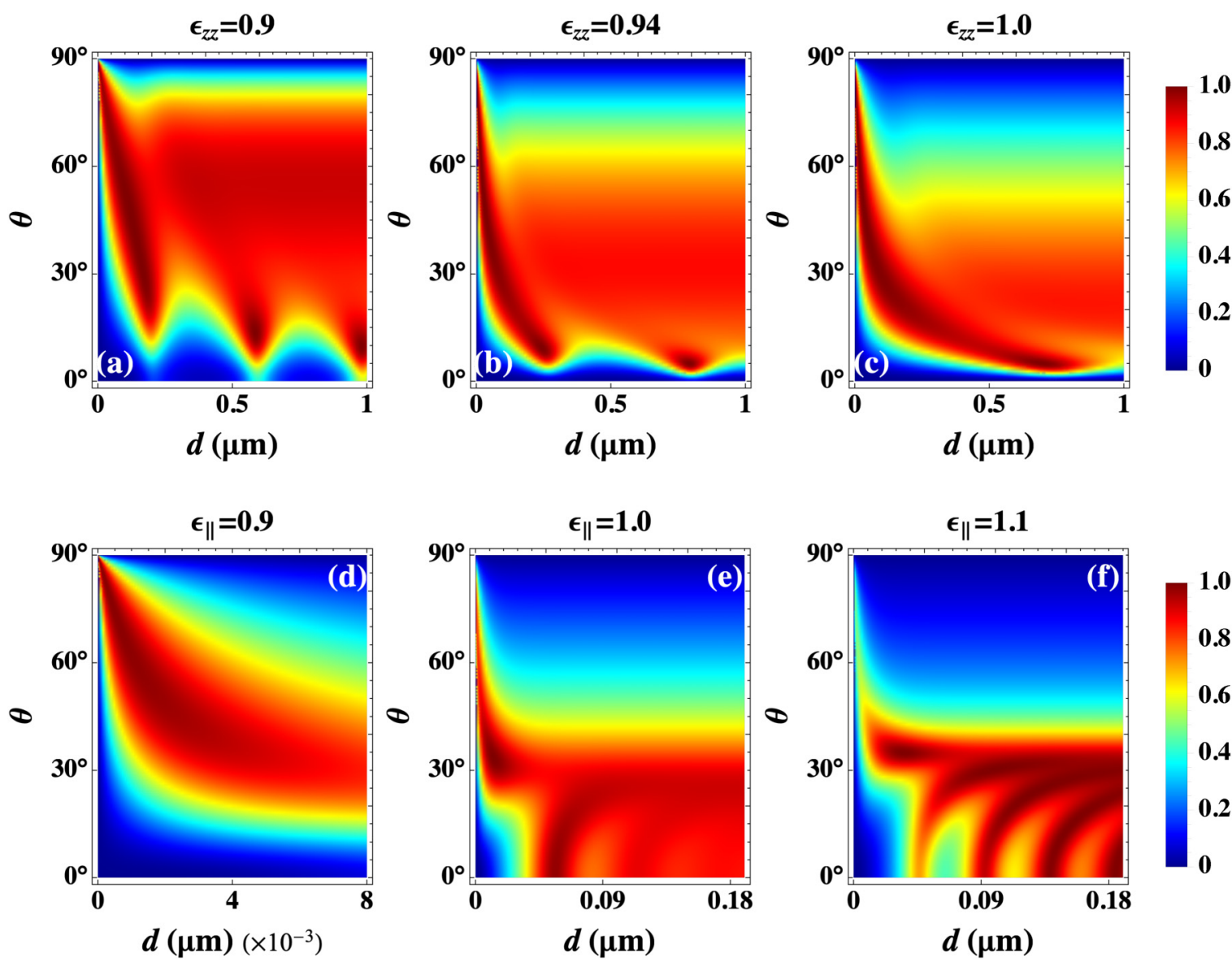

FIG. 5. Color maps representing the absorptance as a function of the angle of incidence and thickness of a black phosphorus film with metallic substrate. Six different strains are considered as labeled. Top row: The parameters used correspond to the strains and frequencies calculated in Figs. 2(a) and 2(b) where there is only out-of-plane strain $\left(\epsilon_{\|}=1\right)$. Bottom row: Only in-plane strain is present $\left(\epsilon_{z z}=1\right)$ and the frequency is set to $\omega=22.6 \mathrm{eV}$.

while for near normal incidence $\left(\theta \sim 0^{\circ}\right)$, thicker layers are required.

We now return to the cases with lateral strain and fixed frequency $\omega=22.6 \mathrm{eV}$. As Fig. 5(d) reveals, when there is a compressive in-plane strain, the high absorption region appears for thin BP layers and a range of incident angles satisfying $\theta \gtrsim 50^{\circ}$. As the strain parameter $\epsilon_{\|}$increases [Figs. 5(e) and 5(f)], the perfect absorption regions become limited to smaller angles of incidence and larger BP layer thicknesses. Correspondingly, there is a substantial increase in the EM modes responsible for complete absorption of the incident wave for tensile strain [Fig. 5(f)], which results in a greater range of permitted $\mathrm{BP}$ thicknesses.

The compressive and tensile strains (of magnitude less than $\lesssim 4 \%$ ) considered throughout the paper present experimentally accessible regimes for fabricating a perfect-absorber device. We have found that a thin spacer layer in between the BP and metallic substrate often has little effect on the results. Therefore, one possible configuration for controlling the in-plane strain of the BP film could involve an elastomeric spacer or matrix containing a BP stack and metal. With proper tailoring of the elastomeric vertical edges, equal strain can be exerted throughout BP layer. For larger strains, we assume that the bulk BP undergoes strain values that do not exceed $10 \%$, thus maintaining the more energetically stable allotrope, consistent with previous theoretical studies [59-61]. Nevertheless, exerting strain values on the order of $10 \%$ can be challenging in practice with current experimental capabilities [61]. For larger systems, the strain may become inhomogeneous under specific situations. In this case, experimental guidance for modeling the explicit spatially inhomogeneous strain pattern seems necessary as there are numerous options to consider. Moreover, this problem goes beyond first-principles calculations as millions of atoms might be involved and other approaches such as empirical potential or effective Hamiltonian treatments should be employed for calculating the dielectric response of such systems. In the next section, we expand the multiscale approach presented above to spatially nonuniform strain patterns.

\section{B. Inhomogeneously strained system}

As is seen above, by modifying the permittivity tensor, an externally applied strain can considerably change the response of a material to an incident electromagnetic wave. If the applied strain within the material is spatially distributed in a nonuniform way, the permittivity tensor becomes spatially inhomogeneous as well. To simulate an inhomogeneously strained system, one should resort to Maxwell's equations with location-dependent permittivity $\overline{\overline{\boldsymbol{\varepsilon}}}_{n}(\mathbf{r})$ and permeability 


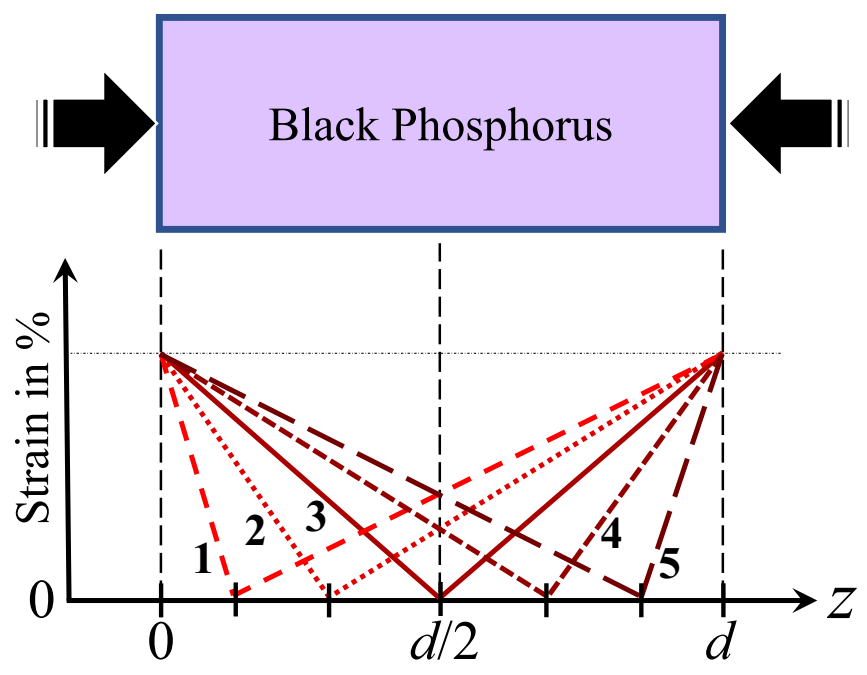

FIG. 6. Inhomogeneous strain in the black phosphorous is accounted for by using a linear $z$-dependent model. The spatially inhomogeneous strain develops along the $z$ direction and it varies linearly (red lines) along the strain direction from a maximum (horizontal dashed line) at locations $z=0$ and $z=d$ to zero strain at points marked by the vertical small indicators at $z=$ $d / 6, d / 3, d / 2,2 d / 3,5 d / 6$. The pertaining curves to these five linear models are marked by $1,2,3,4,5$. The arrows portray the direction of applied strain that propagates linearly inside the BP region confined between $z=0$ and $z=d$.

$\overline{\overline{\boldsymbol{\mu}}}_{n}(\mathbf{r})$ :

$$
\begin{aligned}
& \nabla \times\left(\overline{\overline{\boldsymbol{\mu}}}_{n}^{-1}(\mathbf{r}) \boldsymbol{\nabla} \times \boldsymbol{E}(\mathbf{r})\right)=\omega^{2} \mu_{0} \varepsilon_{0} \overline{\overline{\boldsymbol{\varepsilon}}}_{n}(\mathbf{r}) \boldsymbol{E}(\mathbf{r}), \\
& \nabla \times\left(\overline{\overline{\boldsymbol{\varepsilon}}}_{n}^{-1}(\mathbf{r}) \nabla \times \boldsymbol{H}(\mathbf{r})\right)=\omega^{2} \mu_{0} \varepsilon_{0} \overline{\overline{\boldsymbol{\mu}}}_{n}(\mathbf{r}) \boldsymbol{H}(\mathbf{r}) .
\end{aligned}
$$

The above equations describe the behavior of the electromagnetic fields $\mathbf{E}, \mathbf{H}$ for a generic system with a spatiallyinhomogeneous electromagnetic response. To be able to make use of Eqs. (16), we consider simple linear models for the spatial behavior of the permittivity, as shown in Fig. 6, and the models are marked by $1,2,3,4,5$. The strain takes its maximum value at the interfaces surrounding $\mathrm{BP}(z=0, d)$ and then linearly declines to zero at $z=d / 6, d / 3, d / 2,2 d / 3,5 d / 6$ corresponding to models $1,2,3,4,5$, respectively. By considering the model described above for an inhomogeneous strained system and the fact that BP is a nonmagnetic material, the permeability and permittivity tensors reduce to $\overline{\overline{\boldsymbol{\mu}}}_{n}(\mathbf{r})=\overline{\overline{\mathbf{1}}}$ and $\overline{\overline{\boldsymbol{\varepsilon}}}_{n}(\mathbf{r})=\overline{\overline{\boldsymbol{\varepsilon}}}_{n}(z)$. We consider the same form for the incident electromagnetic field, given by Eqs. (5), in the inhomogeneously strained system as that considered in the previous section for uniformly strained BP. By incorporating the above assumptions into Eq. (16), we arrive at the following differential equation for the $H_{y}$ field:

$$
\begin{aligned}
& \frac{d^{2} H_{y}(z)}{d z^{2}}+\varepsilon_{1 x}(z) \frac{d \varepsilon_{1 x}^{-1}(z)}{d z} \frac{d H_{y}(z)}{d z} \\
& \quad+\left(\omega^{2} \mu_{0} \varepsilon_{0} \varepsilon_{1 x}(z)-\frac{\varepsilon_{1 x}(z)}{\varepsilon_{1 z}(z)} k_{x}^{2}\right) H_{y}(z)=0 .
\end{aligned}
$$

After solving Eq. (17) with the boundary conditions given in Sec. II, the $E_{x}(z), E_{y}(z)$ components of the electric field can be obtained by substituting the $H_{y}(z)$ field into the original Maxwell's equations, i.e., Eqs. (6).

For a sufficiently large BP sample, the local permittivity at a certain location with a certain strain (according to the above linear model) can be approximately calculated with DFT using the bulk black phosphorus unit cell. Following this approach yields the spatial profile for the permittivity $\varepsilon_{1 z}(z)$ shown in Fig. 7. To proceed towards tractable solutions, we assume that the applied strain in the $x$ and $y$ directions is uniform so that the parallel wave-vector component $k_{x}$ remains a conserved quantity upon the scattering process. Note that otherwise, Maxwell's equations (16) result in three-dimensional position-dependent partial differential equations, making any subsequent analysis highly complicated.

Incorporating the linear model for $\varepsilon_{1 z}(z)$ into Eq. (17), we study the absorption properties of this system in Fig. 8. The absorptance $A$ is plotted against the incident angle $\theta$, when the in-plane strain (strain exerted in the $x y$ plane) can have a finite value. In Figs. '8(a), 8(f)', '8(b), 8(g)', '8(c), 8(h)', '8(d), 8(i)', and ' $8(\mathrm{e}), 8(\mathrm{j})$ ', the in-plane strain is set to $-10 \%\left(\epsilon_{\|}=0.90\right)$, $-6 \%\left(\epsilon_{\|}=0.94\right), 0 \%\left(\epsilon_{\|}=1.0\right),-+6 \%\left(\epsilon_{\|}=1.06\right)$, and $+10 \%\left(\epsilon_{\|}=1.10\right)$, respectively. The maximum of strain in the $z$ direction at the boundaries $z=0, d / 2$ are set to a representative value, i.e., $-10 \%$ that linearly declines toward the middle of the BP layer according to the models described in Fig. 6. The top row panels in Fig. 8 illustrate the absorptance when BP possesses a thickness of $d=0.5 \mu \mathrm{m}, 0.3 \mu \mathrm{m}$, and $0.1 \mu \mathrm{m}$ when model 1 is implemented. As is clearly seen, the perfect absorption found in the uniform case [Fig. 4(a)] can also be achieved by manipulating the thickness of the BP layer $d$ in the inhomogeneous model scenario considered. As in the homogenous strain case, the in-plane strain can control the angle of perfect absorption although now limited to $\theta \lesssim 30^{\circ}$. Also, the results reveal that for the linearly inhomogeneous strain model implemented, the system absorbs the incident electromagnetic wave the most when the thickness of the BP layer is around $d=0.3 \mu \mathrm{m}$. In the bottom row panels of Fig. 8, the thickness of the BP layer is set to $d=0.3 \mu \mathrm{m}$ and the five different strain models shown in Fig. 6 are implemented. The results show that the different inhomogeneous strain models keep the perfect absorption of the device almost intact. Although not shown, it was found that the multiple perfect absorption peaks found for the $\epsilon_{\|}=1.1$ cases of tensile strain in Fig. 4(a) reduces as the modified effective dielectric response increases the BP reflectivity due to the incident wave not fully coupling to the structure. Therefore, by tailoring the thickness of the BP appropriately, perfect absorption can also be achieved when there is a spatially inhomogeneous strain-dependent dielectric response.

\section{CONCLUSION}

We have studied the absorption of electromagnetic energy for a semi-infinite strained bulk black phosphorus layer that is deposited on a metallic substrate. Using the density functional theory of electronic structure, we obtained the dielectric response tensor of black phosphorus subject to compressive and tensile strains along principal crystallography directions. 

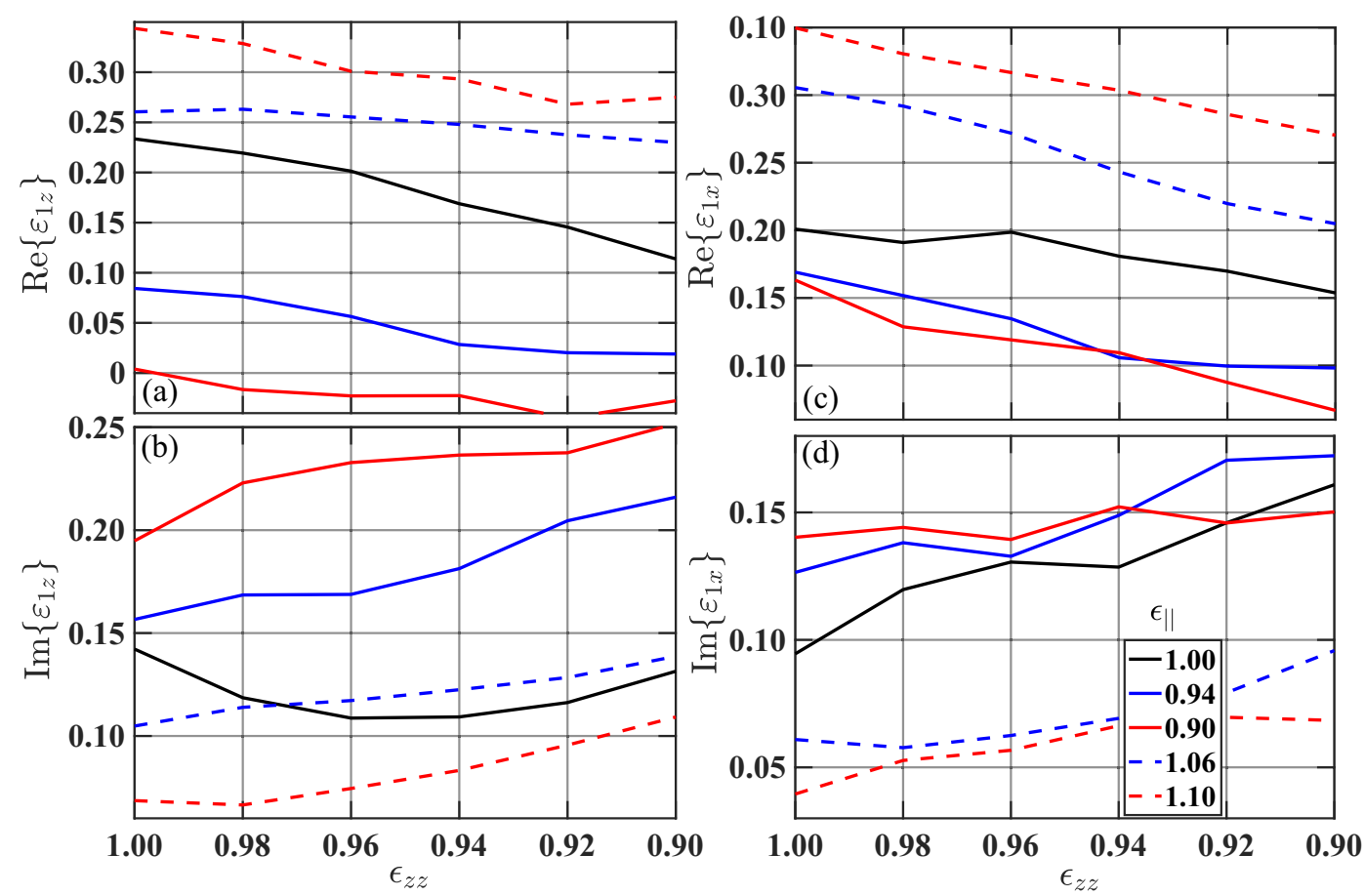

FIG. 7. The permittivity of BP as a function of compressive strain $\epsilon_{z z}$ from 0 to $-10 \%$. The panels (a) and (b) show the real and imaginary parts of $\varepsilon_{1 z}$ whereas the panels (c) and (d) are the real and imaginary part of $\varepsilon_{1 x}$. The in-plane strain spans the strain-free, compressive, and tensile strain regimes; $\epsilon_{\|}=1.0,0.94,0.90,1.06,1.10$.

The permittivity along the direction normal to the black phosphorus layer was found to exhibit multiple epsilon-near-zero conditions in a large frequency range by applying appropriate strain. Incorporating the calculated permittivity tensor, we solved Maxwell's equations for the electromagnetic modes, demonstrating that the exertion of strain can switch the direction of electromagnetic wave energy flow within the black phosphorus layer. The applied strain was demonstrated as an effective control knob for tuning the optical and electronic properties of black phosphorus, resulting in efficient control
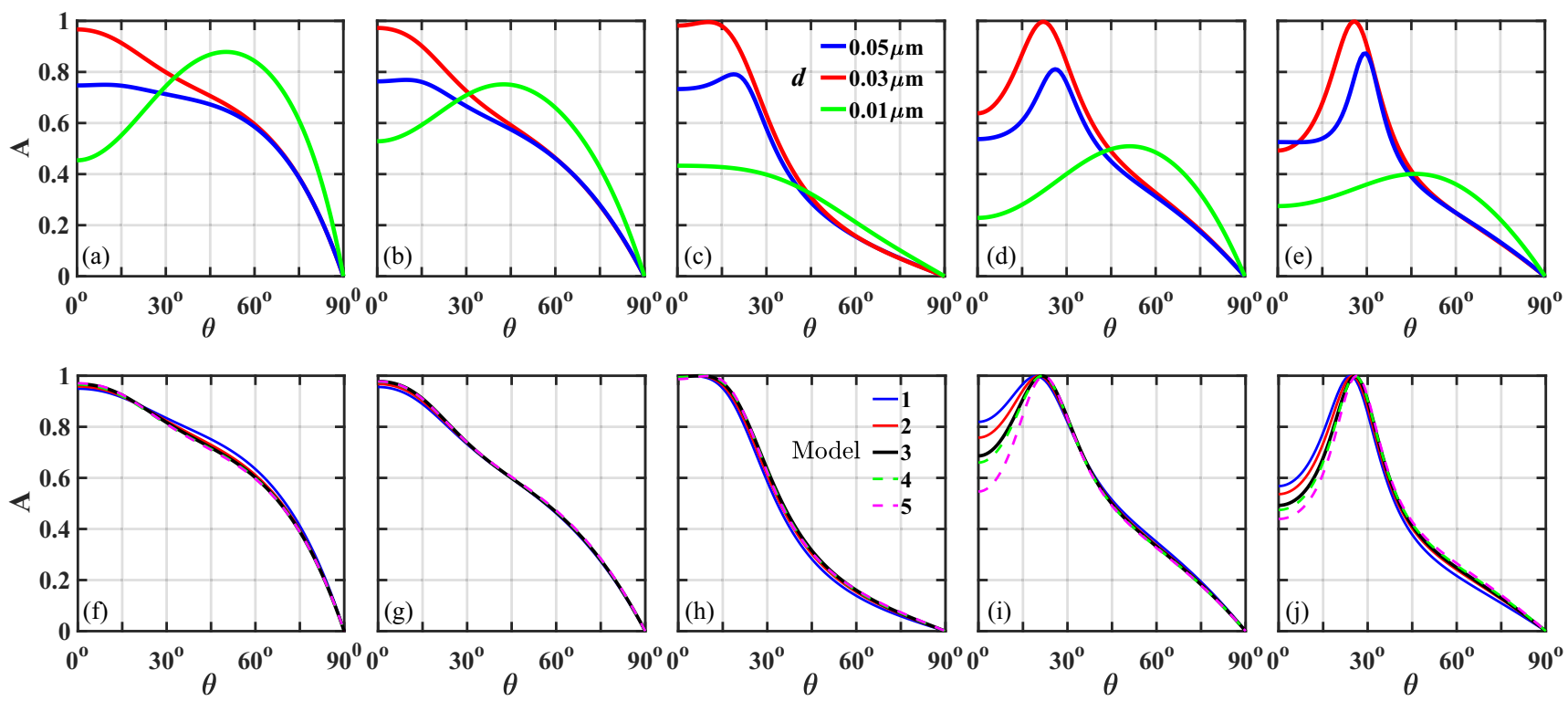

FIG. 8. Absorptance vs the incident angle $\theta$ of electromagnetic wave. The frequency of the electromagnetic wave is set to $\omega=22.6 \mathrm{eV}$. The in-plane strain increases from the leftmost column to the rightmost column, i.e., $\epsilon_{\|}=-10 \%,-6.0 \%, 0.0 \%,+6.0 \%,+10 \%$ in $(\mathrm{a})$, (f), (b), (g), (c),(h), (d),(i), and (e),(j), respectively. In the top row panels, three different thicknesses of BP are set; $d=0.5 \mu \mathrm{m}, 0.3 \mu \mathrm{m}, 0.1 \mu \mathrm{m}$, when model 1 is implemented. In the bottom row panels, the thickness of BP is set to $d=0.3 \mu$ and the five different models (marked by $1,2,3,4,5)$ are implemented. 
of the absorption of an incident electromagnetic wave with a largely tunable angle. Considering spatially nonuniform strain profiles along the direction normal to the black phosphorus layer, we showed that the application of strain can produce near perfect absorption of the incident wave. The presented results open up avenues for the practical use of coherent and perfect absorption over a wide range of incident angles, frequencies, and layer thicknesses.

\section{ACKNOWLEDGMENTS}

The DFT calculations were performed using the resources provided by UNINETT Sigma2 - the National Infrastructure for High Performance Computing and Data Storage in Norway. Part of the calculations were performed using HPC resources from the DOD High Performance Computing Modernization Program (HPCMP). K.H. is supported in part by the NAWCWD In Laboratory Independent Research (ILIR) program and a grant of HPC resources from the DOD HPCMP.

\section{APPENDIX A: FIELD IMPEDANCE-MATCHING AND WAVEGUIDE MODE APPROACHES FOR COHERENT PERFECT ABSORPTION}

To gain further insight into the absorption mechanism occurring in the BP structure, we consider alternative approaches that can reveal the underlying EM modes responsible for the complete absorption of incident EM energy. In the case of coherent perfect absorption, the metallic layer must generate the proper reflective waves that interfere destructively in the BP region. To isolate the absorption effects that arise from purely dissipative processes, we have considered in this paper moderate to extremely weak amounts of loss. As was previously shown, when the structure exhibits an ENZ response and the component of the permittivity tensor perpendicular to BP layer exhibits low loss, relatively thin layers are required to achieve complete absorption [43]. To discuss the previous findings in Fig. 5 within the context of coherent perfect absorption, we implement two independent and complimentary methods.

The first method involves matching the field impedance of the incident plane wave to that of the $\mathrm{BP} /$ metal structure. This is achieved by setting $\mathcal{Z}_{0}=\mathcal{Z}_{1}$ at the vacuum/BP interface, where the field impedance is defined as

$$
\mathcal{Z}_{i}=\left.\frac{E_{x i}}{H_{y i}}\right|_{z=0},
$$

for either the vacuum or BP regions (identified by $i=0,1$ ). For the $\mathrm{BP} /$ metal system, we find

$$
\mathcal{Z}_{1}=-i \eta_{0} \frac{\left(\epsilon_{1 z} k_{0}^{2}-k_{0 x}^{2}\right) \tan \left(k_{1 z} d\right)}{\epsilon_{1 z} k_{0} k_{1 z}},
$$

whereas for the vacuum region the result is simply $\mathcal{Z}_{0}=$ $\eta_{0} k_{0 z} / k_{0}$. After impedance matching, the resultant expression constrains the allowed geometrical and material parameters leading to the reflection coefficient $r$ vanishing [see Eq. (10)].

An alternative approach views the structure in Fig. 1 as a waveguide, so that the incident plane wave is absent and the

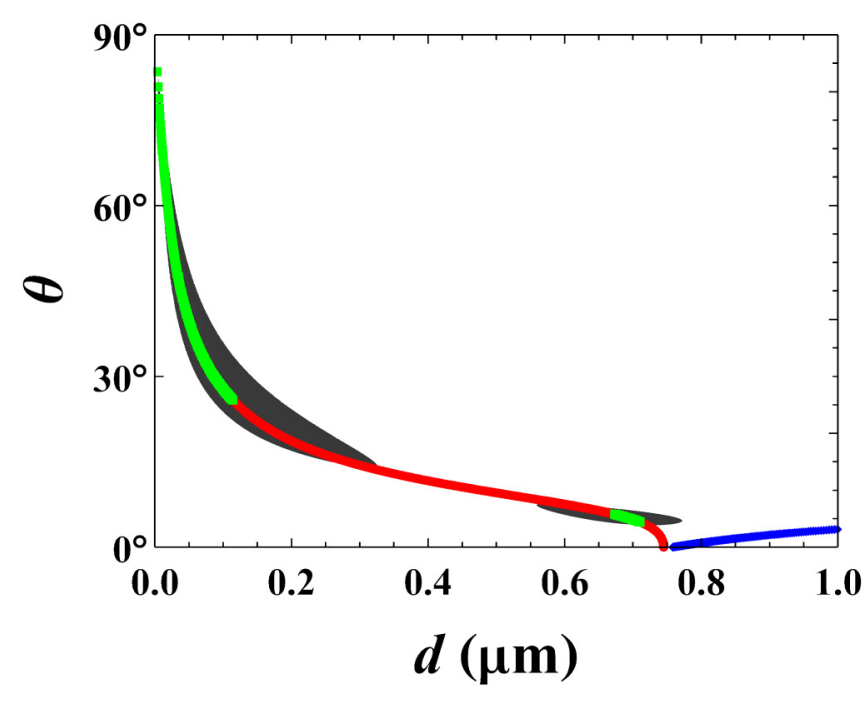

FIG. 9. Electromagnetic modes for the system in Fig. 1 with the same parameter values as used in Fig. 5(c). The red curve depicts the fast-wave $\left(k_{0 x} / k_{0}<1\right)$ waveguide modes, while the green curves correspond to the impedance matched modes (overlapping with the red curve). As the thickness increases, eventually radiative leaky waves emerge (blue curve). The angle $\theta$ is determined from the propagation constants $k_{0 x}$ via $\theta=\arcsin \left(k_{0 x} / k_{0}\right)$. For reference, the black region is extracted from the high absorptance ( $>95 \%)$ data of Fig. 5(c).

electric and magnetic fields in the vacuum region decay exponentially. For example, the magnetic field would be written $H_{y 0} \sim e^{-k_{0 z} z} e^{i k_{0 x} x}$. As before, the form for the EM fields in the $\mathrm{BP}$ region are linear combinations of waves with the wave vectors given in Eq. (8). After invoking the usual interface and boundary conditions, the inherent guided wave modes of the structure can be found. The result is Eq. (15), which is equivalent to finding where the denominator of the reflection coefficient in Eq. (10) vanishes. Since we are interested in perfect coupling of the incident plane wave to the waveguide, we focus on the fast-wave nonradiative solutions whereby $k_{1 z} / k_{0}<1$.

The results of the two approaches are presented in Fig. 9, where the calculated perfect absorption modes of BP layered structure are plotted. All the parameter values used are identical to those of Fig. 5(c). The invariant wave-vector component $k_{0 x}$ is varied along with $d$, and each data point is an allowed root to the corresponding transcendental equations. The green curves are the calculated waveguide modes [Eq. (15)], using a root-finding algorithm, and the red curves arise from the field impedance matching method. As the thickness increases, leaky wave modes arise where the solutions to Eq. (15) admit propagation constants with a finite imaginary component $\alpha>0$. As can be seen, the results of the two methods are in excellent agreement.

\section{APPENDIX B: DFT SIMULATIONS OF UNIT CELL PARAMETERS, ELECTRONIC BAND STRUCTURE, AND DENSITY OF STATES}

The lattice parameters $\left(a_{0}, b_{0}, c_{0}, \alpha, \beta, \gamma\right)$ and the location of $\mathrm{AB}$ stacking order for phosphorus atoms $(x, y, z)$ in an 
TABLE III. Lattice parameters $\left(a_{0}, b_{0}, c_{0}, \alpha, \beta, \gamma\right)$ for black phosphorus undergoing in-plane and out-of-plane compressive and tensile strains.

\begin{tabular}{lcccc}
\hline \hline & $a_{0}(\AA)$ & $b_{0}(\AA)$ & $c_{0}(\AA)$ & $\operatorname{Vol}\left(\AA^{3}\right)$ \\
\hline In plane & & & & \\
10\% Compressive & 2.98431 & 4.05576 & 10.44520 & 126.424987 \\
10\% Tensile & 3.64749 & 4.95704 & 10.44520 & 188.857084 \\
4\% Compressive & 3.18326 & 4.32614 & 10.44520 & 143.843231 \\
4\% Tensile & 3.44854 & 4.68666 & 10.44520 & 168.816718 \\
Out of plane & & & & \\
10\% Compressive & 3.31590 & 4.50640 & 9.40065 & 140.471775 \\
10\% Tensile & 3.31590 & 4.50640 & 11.48970 & 171.687978 \\
4\% Compressive & 3.31590 & 4.50640 & 10.02740 & 149.837157 \\
4\% Tensile & 3.31590 & 4.50640 & 10.86300 & 162.323337 \\
& $\alpha(\mathrm{deg})$ & $\beta(\mathrm{deg})$ & $\gamma(\mathrm{deg})$ & \\
& $90^{\circ}$ & $90^{\circ}$ & $90^{\circ}$ & \\
\hline \hline
\end{tabular}

unstrained unit cell are summarized in Table II. To simulate strained BP, we have scaled both the unit cell and atomic locations (in percent, with respect to the unstrained parameters), depending on the type of strain applied. To characterize the material response to strain, we also list the in-plane covalent bonds $\left(\ell_{1}\right)$, interlayer distance $\left(\ell_{2}\right)$, and in-plane dihedral angle $(\Gamma)$ for tensile and compressive $10 \%$ in-plane strains:

$\begin{array}{cccc} & \text { compressive } & \text { unstrained } & \text { tensile } \\ \ell_{1}(\AA) & 2.00521 & 2.22802 & 2.45082 \\ \ell_{2}(\AA) & 2.23537 & 2.26009 & 2.28711 \\ \Gamma(\mathrm{deg}) & 27.0833 & 29.9636 & 32.8056\end{array}$

In Table III, we summarize the lattice parameters for BP when it is subject to in-plane or out-of-plane compressive and tensile strains. Note that the normalized positions of the atoms are identical to those given in Table II.

We present in Fig. 10 the calculations for the electronic band structure (left panels) and total density of states (TDOS) (right panels) for bulk black phosphorus with both in-plane and out-of-plane compressive and tensile stresses. From the top row, it is evident that the band gap is sensitive to the inplane strain and whether it is of the compressive or tensile type. Importantly, there are no band crossings along any of the symmetry points in Fig. 10(c), and due to the applied tensile strain a clear gap emerges at the Fermi energy. Upon compressive strain, the interatomic spacing is reduced, the gap vanishes, and the system becomes conductive. Depending on the applied strain, the band structure exhibits an increasing number of interband transitions leading to increased losses. The opposite occurs when tensile forces are applied, and the corresponding modified lattice constants lead to a band gap and changing dielectric optical properties. This is consistent with the top row of Fig. 2, where it was shown that as BP experiences increased compressive strain ( $\epsilon_{z z}$ decreases), there is a greater dissipation as seen in the increased imaginary component of the permittivity. Similar behavior is seen at these frequencies when there is in-plane strain (not shown).

The tunability of the band gap of BP is very well understood $[64,65]$. As can be seen in Fig. 10(a), the DFT-predicted band gap of bulk BP is zero while the experimental optical band gap is $0.3 \mathrm{eV}$. In effect, this discrepancy originates from the underestimation of the BP band gap by standard DFT functionals, such as PBE. To improve the band gap prediction, one may either repeat the calculation by a hybrid functional or resort to the $\mathrm{GW}$ approximation for the contribution of self-energy [62-65]. While GW has a more solid fundamental footing, DFT provides better agreement with experimental band gaps for some material systems. For example, in the

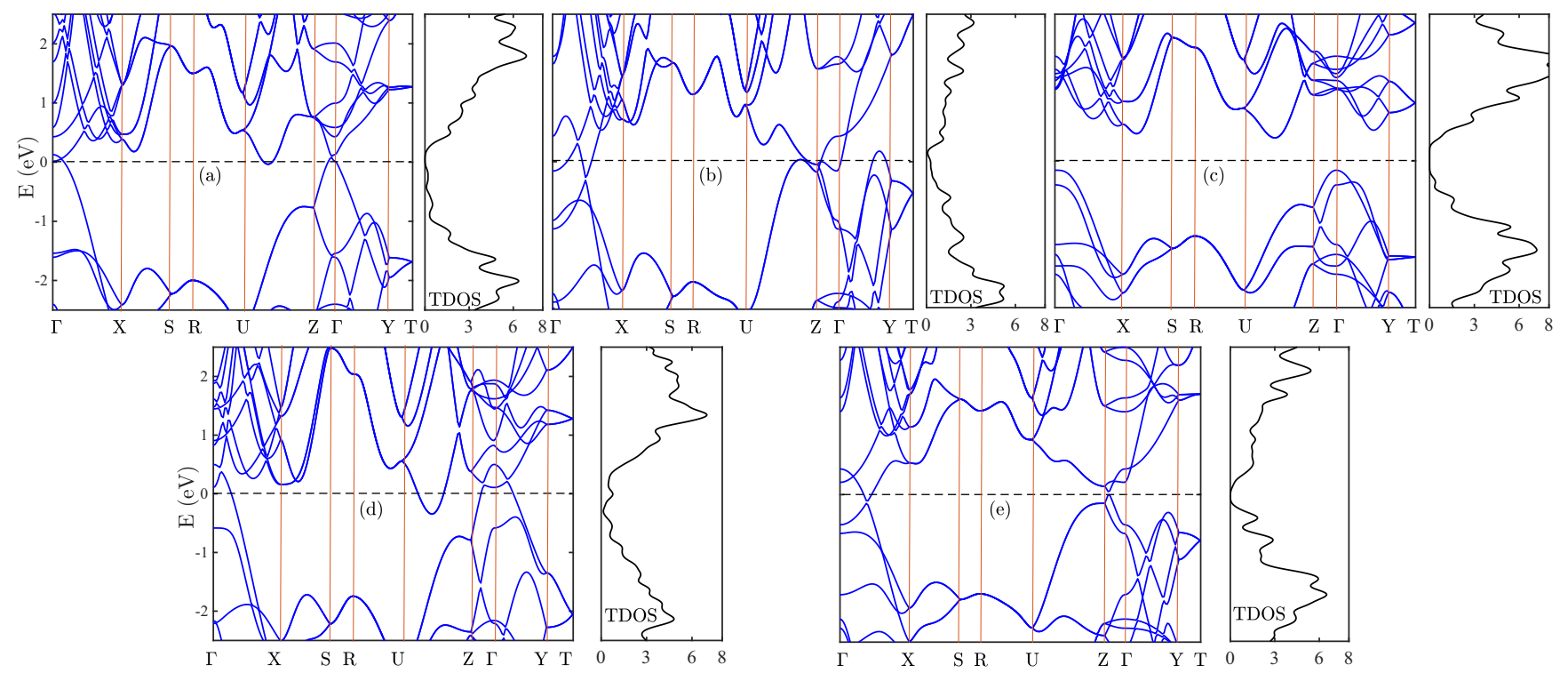

FIG. 10. Electronic band structures and total densities of states for bulk black phosphorus. The strain parameters are as follows: (a) $\epsilon_{\|}=1.0$, (b) $\epsilon_{\|}=0.9$, and (c) $\epsilon_{\|}=1.1$ for in-plane strain and (d) $\epsilon_{z z}=0.9$ and (e) $\epsilon_{z z}=1.1$ for out-of-plane strain. In (a)-(c) the system is unstrained along $z\left(\epsilon_{z z}=1\right)$, and there is no in-plane strain in (d) and (e) $\left(\epsilon_{\|}=1.0\right)$. The Fermi energy is located at the energy $E=0$. The axes labels of the top row panels are the same as for the bottom row panels. 

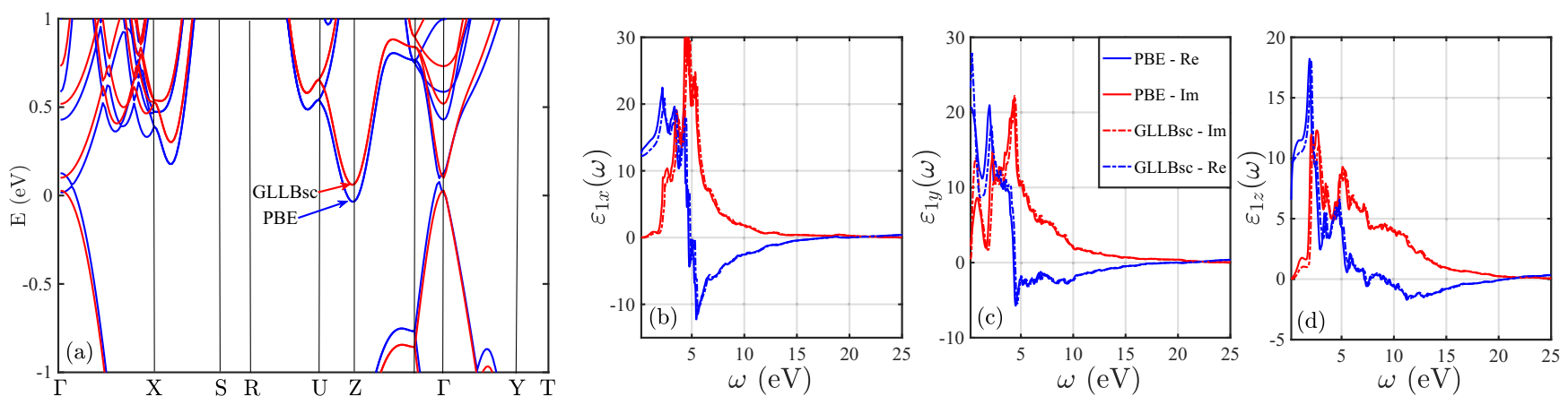

FIG. 11. Band structure and permittivity components for unstrained BP using PBE and GLLBsc functionals. (a) The band structure is shown for PBE and GLLBsc by the blue and red curves, respectively. (b),(c) Plots of the permittivity tensor components $\left(\varepsilon_{1 x, 1 y, 1 z}\right)$ as a function frequency. The solid and dashed curves show PBE and GLLBsc results, respectively. The real (Re) and imaginary (Im) parts are displayed by the blue and red colors, respectively.

case of single-layer $\mathrm{MoS}_{2}$, DFT-PBE and DFT-HSE (hybrid functional) yield reasonable values $(1.6 \mathrm{eV}$ and $1.9 \mathrm{eV}$, respectively) [66], very close to the photoluminescence (PL) experimental value $(1.8-1.9 \mathrm{eV})[67,68]$. Conversely, the band gap of single layer $\mathrm{MoS}_{2}$ is seriously overestimated by the GW method $(2.7 \mathrm{eV})$ [66]. It is well known that exciton effects [69], absent in standard GW calculations, lead to a lower effective band gap as found in PL experiments. Hence, in some cases DFT methods, in particular based on hybrid exchange-correlation functional approximations, can lead to band gaps that fit experimental results better than those for GW.

To illustrate that the application of different functionals can improve the band gap underestimation by PBE while providing negligible difference in linear response, we have plotted the band structure and the corresponding components of the permittivity tensor for unstrained BP using both the PBE and GLLBsc functionals [70,71] in Fig. 11. As is clearly seen in Fig. 11(a), the (indirect) band gap at the $\Gamma$ and $Z$ points opens up to $\sim 0.1 \mathrm{eV}$ for GLLBsc. A comparison of the permittivity tensor components in Figs. 11(b)-11(c) displays only slight modifications between PBE and GLLBsc. This can be understood by the fact that the dielectric response is a collective response, meaning that momentum space is integrated out within the Brillouin zone. Therefore, slight shifts of electronic bands do not severely alter the dielectric response. Consequently, by simply looking at DOS or band structure it is impractical to make a conclusion about the behavior or the dielectric response of BP. In any case, one should note that the random phase approximation used for calculating the dielectric response tensor does not include exchange-correlation contributions (although there is surely a dependence via the predetermined ground-state electron density) [45]. We emphasize that the presented results and conclusions made for the absorption in BP-based heterostructures rely only on the ENZ mechanism in the low-dissipation regime. Our calculations reveal that several ENZ modes are accessible throughout the frequency interval, and any possible band gap corrections will not affect the main message of this work. To demonstrate this fact, we have plotted in Fig. 12 the angle-dependent absorptance by using both the PBE and GLLBsc functionals. The dashed and solid curves correspond to the GLLBsc and PBE functionals, respectively. Note that all parameters are identical to those used in Fig. 4(a). As seen, the use of the different functionals results in negligible variations in absorptance.

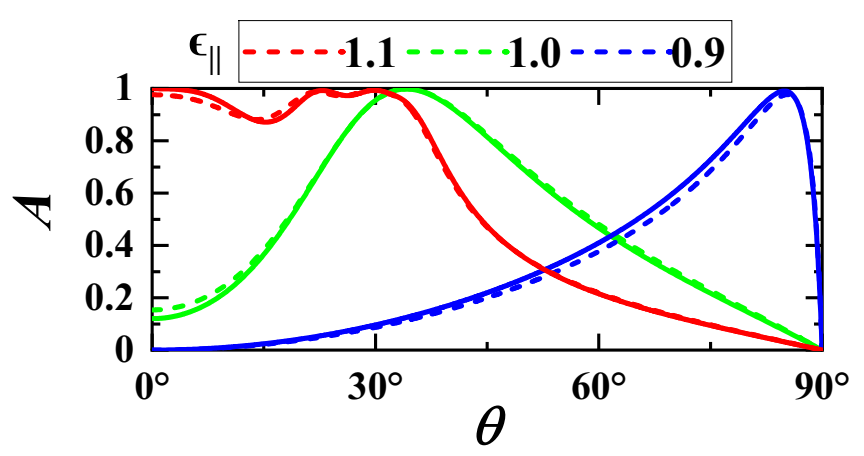

FIG. 12. Absorptance $(A)$ vs the incident angle $\theta$ for three inplane strain values $\epsilon_{\|}=1.1,1.0,0.9$. The frequency of the incident wave is set at $\omega=22.6 \mathrm{eV}$. The corresponding permittivity components of the dashed and solid lines are obtained using the GLLBsc and PBE functionals, respectively.
[1] A. S. Rodin, A. Carvalho, and A. H. C. Neto, Strain-Induced Gap Modification in Black Phosphorous, Phys. Rev. Lett. 112, 176801 (2014).

[2] X. Peng, Q. Wei, and A. Copple, Strain-engineered direct-indirect band gap transition and its mechanism in two-dimensional phosphorene, Phys. Rev. B 90, 085402 (2014).
[3] L. Voon, A. Lopez-Bezanilla, J. Wang, Y. Zhang, M. Willatzen, Effective Hamiltonians for phosphorene and silicene, New J. Phys. 17, 025004 (2015).

[4] L. Voon, J. Wang, Y. Zhang, and M. Willatzen, Band parameters of phosphorene, J. Phys.: Conf. Ser. 633, 012042 (2015).

[5] Y. Wei, F. Lu, T. Zhou, X. Luo, and Y. Zhao, Stacking sequences of black phosphorous allotropes and the corresponding 
few-layer phosphorenes, Phys. Chem. Chem. Phys. 20, 10185 (2018).

[6] T. Fang, T. Liu, Z. Jiang, R. Yang, P. Servati, and G. Xia, Fabrication and the Interlayer Coupling Effect of Twisted Stacked Black phosphorous for Optical Applications, ACS Appl. Nano Mater. 2, 3138 (2019).

[7] Z. Zhang, L. Li, J. Horng, N. Z. Wang, F. Yang, Y. Yu, Y. Zhang, G. Chen, K. Watanabe, T. Taniguchi, X. H. Chen, F. Wang, and Y. Zhang, Strain-modulated bandgap and piezo-resistive effect in black phosphorous field-effect transistors, Nano Lett. 17, 6097 (2017).

[8] S. Das, W. Zhang, M. Demarteau, A. Hoffmann, M. Dubey, and A. Roelofs, Tunable transport gap in phosphorene, Nano Lett. 14, 5733 (2014).

[9] Z. Qin, G. Xie, H. Zhang, C. Zhao, P. Yuan, S. Wen, and L. Qian, Black phosphorous as saturable absorber for the Qswitched Er:ZBLAN fiber laser at $28 \mu \mathrm{m}$, Opt. Express 23, 24713 (2015).

[10] M. Alidoust, M. Willatzen, A.-P. Jauho, Strain-engineered Majorana zero energy modes and $\varphi_{0}$ Josephson state in black phosphorous, Phys. Rev. B 98, 085414 (2018).

[11] M. Alidoust, M. Willatzen, A.-P. Jauho, Fraunhofer response and supercurrent spin switching in black phosphorous with strain and disorder, Phys. Rev. B 98, 184505 (2018).

[12] M. Alidoust, M. Willatzen, A.-P. Jauho, Control of superconducting pairing symmetries in monolayer black phosphorous, Phys. Rev. B 99, 125417 (2019).

[13] Y. Ren, P. Liu, F. Cheng, and G. Zhou, Strain-induced effects in zigzag-edged blue phosphorene nanoribbons with edge sulfur passivation, J. Phys.: Condens. Matter 30, 395303 (2018).

[14] D. Odkhuu, D. Sangaa, and P. Taivansaikhan, Strain tunable spin reorientation of an individual Fe atom on 2D blue phosphorous, J. Phys.: Condens. Matter 31, 485802 (2019).

[15] W. Li, F. Cheng, Anisotropy transport in monolayer black phosphorous under period magnetic modulation, Physica E 114, 113631 (2019).

[16] D. Pan, T. C. Wang, W. Xiao, D. Hu and Y. Yao, Simulations of twisted bilayer orthorhombic black phosphorus, Phys. Rev. B 96, 041411(R) (2017).

[17] D. Pan, C. Liu, G. B. Liu, S. Feng and Y. Yao, Physical Fingerprints of the $2 \mathrm{O}-\mathrm{t} \alpha \mathrm{P}$ Phase in Phosphorene Stacking, J. Phys. Chem. Lett. 10, 3190 (2019).

[18] T. Guo and C. Argyropoulos, Tunable and broadband coherent perfect absorption by ultrathin black phosphorous metasurfaces, J. Opt. Soc. Am. B 36, 2962 (2019).

[19] D. David, C. Godet, F. Johansson, and A. Lindblad, Quantitative analysis of plasmon excitations in hard x-ray photoelectron spectra of bulk black phosphorous, Appl. Surf. Sci. 505, 144385 (2019).

[20] T. Liu, X. Jiang, C. Zhou, and S. Xiao, Black phosphorousbased anisotropic absorption structure in the mid-infrared, Opt. Express 27, 27618 (2019).

[21] S. Xiao, T. Liu, L. Cheng, C. Zhou, X. Jiang, Z. Li, and C. Xu, Tunable Anisotropic Absorption in Hyperbolic Metamaterials Based on Black Phosphorous/Dielectric Multilayer Structures, J. Lightwave Technol. 37, 3290 (2019).

[22] S. Zhang, Y. Huang, Y. Liu, Y. Shao, C. Fang, G. Han, J. Zhang, and Y. Hao, Multiple-layer black phosphorous phototransistor with Si microdisk resonator based on whispering gallery modes, Appl. Opt. 58, 4400 (2019).
[23] P. T. T. Le, K. Mirabbaszadeh and M. Yarmohammadi, Blue shift in the interband optical transitions of gated monolayer black phosphorous, J. Appl. Phys. 125, 193101 (2019).

[24] D. Dong, Y. Liu, Y. Fei, Y. Fan, J. Li, Y. Feng, and Y. Fu, Designing a nearly perfect infrared absorber in monolayer black phosphorous, Appl. Opt. 58, 3862 (2019).

[25] N. Feng, J. Zhu, C. Li, Y. Zhang, Z. Wang, Z. Liang, and Q. H. Liu, Near-unity anisotropic infrared absorption in monolayer black phosphorous with/without subwavelength patterning design, IEEE 25, 4700407 (2019).

[26] Y. Huang, X. Liu, Y. Liu, Y. Shao, S. Zhang, C. Fang, G. Han, J. Zhang, and Y. Hao, Nanostructured multiple-layer black phosphorous photodetector based on localized surface plasmon resonance, Opt. Mater. Express 9, 739 (2019).

[27] W. Shen, C. Hu, S. Huo, Z. Sun, G. Fan, J. Liu, L. Sun and X. $\mathrm{Hu}$, Black phosphorous Nano-Polarizer with High Extinction Ratio in Visible and Near-Infrared Regime, Nanomaterials $\mathbf{9}$, 168 (2019).

[28] D. Q. Khoa, M. Davoudiniya, B. D. Hoi and M. Yarmohammadi, Strain engineering of optical activity in phosphorene, RSC Advances 9, 19006 (2019).

[29] Y. M. Qing, H. F. Ma, and T. J. Cui, Tailoring anisotropic perfect absorption in monolayer black phosphorous by critical coupling at terahertz frequencies, Opt. Express 26, 32442 (2018).

[30] C. Fang, Y. Liu, G. Han, Y. Shao, J. Zhang, and Y. Hao, Localized plasmon resonances for black phosphorous bowtie nanoantennas at terahertz frequencies, Opt. Express 26, 27683 (2018).

[31] Q. Hong, F. Xiong, W. Xu, Z. Zhu, K. Liu, X. Yuan, J. Zhang, and S. Qin, Towards high performance hybrid twodimensional material plasmonic devices: strong and highly anisotropic plasmonic resonances in nanostructured grapheneblack phosphorous bilayer, Opt. Express 26, 22528 (2018).

[32] X. Wang, Q. Ma, L. Wu, J. Guo, S. Lu, X. Dai, and Y. Xiang, Tunable terahertz/infrared coherent perfect absorption in a monolayer black phosphorous, Opt. Express 26, 5488 (2018).

[33] J. Wang and Y. Jiang, Infrared absorber based on sandwiched two- dimensional black phosphorous metamaterials, Opt. Express 25, 5206 (2017).

[34] J. Wang, Y. Jiang, and Z. Hu, Dual-band and polarizationindependent infrared absorber based on two-dimensional black phosphorous metamaterials, Opt. Express 25, 22149 (2017).

[35] F. Xiong, J. Zhang, Z. Zhu, X. Yuan, and S. Qin, Strong anisotropic perfect absorption in monolayer black phosphorous and its applica- tion as tunable polarizer, J. Opt. 19, 075002 (2017).

[36] D. Li, H. Jussila, L. Karvonen, G. Ye, H. Lipsanen, X. Chen, and Z. Sun, Polarization and thickness dependent absorption properties of black phosphorous: new saturable absorber for ultrafast pulse generation, Sci. Rep. 5, 15899 (2015).

[37] M. Engel, M. Steiner, and P. Avouris, Black phosphorous photodetector for multispectral, high-resolution imaging, Nano Lett. 14, 6414 (2014).

[38] H. Wang, X. Wang, F. Xia, L. Wang, H. Jiang, Q. Xia, M. L. Chin, M. Dubey, and S. Han, Black phosphorous radiofrequency transistors, Nano Lett. 14, 6424 (2014).

[39] M. N. Gjerding, M. Pandey and K. S. Thygesen, Band structure engineered layered metals for low-loss plasmonics, Nat. Commun. 8, 1 (2017). 
[40] M. Silveirinha and N. Engheta, Tunneling of Electromagnetic Energy through Subwavelength Channels and Bends using $\epsilon$ Near-Zero Materials, Phys. Rev. Lett. 97, 157403 (2006).

[41] H. Galinski, G. Favraud, H. Dong, J. S. T. Gongora, G. Favaro, M. Dbeli, R. Spolenak, A. Fratalocchi, and F. Capasso, Scalable, ultra-resistant structural colors based on network metamaterials, Light: Sci. Appl. 6, e16233 (2017).

[42] D. C. Adams, S. Inampudi, T. Ribaudo, D. Slocum, S. Vangala, N. A. Kuhta, W. D. Goodhue, V. A. Podolskiy, and D. Wasserman, Funneling Light through a Subwavelength Aperture with Epsilon-Near-Zero Materials, Phys. Rev. Lett. 107, 133901 (2011).

[43] S. Feng and K. Halterman, Coherent perfect absorption in epsilon-near-zero metamaterials, Phys. Rev. B 86, 165103 (2012).

[44] M. S. Hybertsen and S. G. Louie, Ab initio static dielectric matrices from the density-functional approach. I. Formulation and application to semiconductors and insulators, Phys. Rev. B 35, 5585 (1987).

[45] D. Pines and P. Nozi 'eres, The theory of quantum liquids (Benjamin, New York, 1966).

[46] J. J. Mortensen, L. B. Hansen, K. W. Jacobsen, Real-space grid implementation of the projector augmented wave method, Phys. Rev. B 71, 035109 (2005).

[47] J. Enkovaara, C. Rostgaard, J. J. Mortensen, J. Chen, M. Dułak, L. Ferrighi, J. Gavnholt, C. Glinsvad, V. Haikola, H. A. Hansen et al., Electronic structure calculations with GPAW: a real-space implementation of the projector augmented-wave method, J. Phys.: Condens. Matter 22, 253202 (2010).

[48] M. Neviere and P. Vincent, Brewster phenomena in a lossy waveguide used just under the cut-off thickness, J. Opt. 11, 153 (1980).

[49] T. S. Luk, S. Campione, I. Kim, S. Feng, Y. C. Jun, S. Liu, J. B. Wright, I. Brener, P. B. Catrysse, S. Fan, and M. B. Sinclair, Directional perfect absorption using deep subwavelength lowpermittivity films, Phys. Rev. B 90, 085411 (2014).

[50] K. Halterman, S. Feng, and V. C. Nguyen, Controlled leaky wave radiation from anisotropic epsilon near zero metamaterials, Phys. Rev. B 84, 075162 (2011).

[51] K. Halterman, M. Alidoust, and A. Zyuzin, Epsilon-near-zero response and tunable perfect absorption in Weyl semimetals, Phys. Rev. B 98, 085109 (2018).

[52] T. Tamaya, T. Kato, K. Tsuchikawa, S. u Konabe, and S. Kawabata, Surface plasmon polaritons in thin-film Weyl semimetals, J. Phys.: Condens. Matter 31, 305001 (2019).

[53] K. Sonowal, A. Singh, and A. Agarwal, Giant optical activity and Kerr effect in type-I and type-II Weyl semimetals, Phys. Rev. B 100, 085436 (2019).

[54] S. Almutairi, Q. Chen, M. Tokman, and A. Belyanin, Four-wave mixing in Weyl semimetals, Phys. Rev. B 101, 235156 (2020).

[55] K. Halterman and M. Alidoust, Waveguide modes in Weyl semimetals with tilted dirac cones, Opt. Express 27, 36164 (2019).
[56] S. Liu, Y. Shou, X. Zhou, W. Cheng, and Z. Luo, Latticedependent spin Hall effect of light in a Weyl semimetal, Opt. Express 28, 10783 (2020).

[57] K. Tsuchikawa, S. Konabe, T. Yamamoto, and S. Kawabata, Characterization of a Weyl semimetal using a unique feature of surface plasmon polaritons, Phys. Rev. B 102, 035443 (2020).

[58] Q. Chen, M. Erukhimova, M. Tokman, and A. Belyanin, Optical Hall effect and gyrotropy of surface polaritons in Weyl semimetals, Phys. Rev. B 100, 235451 (2019).

[59] J. Guan, W. Song, L. Yang, and D. Tomanek, Strain-controlled fundamental gap and structure of bulk black phosphorus, Phys. Rev. B 94, 045414 (2016).

[60] G. Yang, T. Ma, and X. Peng, Superior mechanical flexibility and strained-engineered direct-indirect band gap transition of green phosphorene, Appl. Phys. Lett. 112, 241904 (2018).

[61] X. Li, J. Sun, P. Shahi, M. Gao, A. H. MacDonald, Y. Uwatoko, T. Xiang, J. B. Goodenough, J. Cheng, and J. Zhou, Pressureinduced phase transitions and superconductivity in a black phosphorus single crystal, Nat. Acad. Sci. 115, 9935 (2018).

[62] C. Adamo and V. Barone, Toward reliable density functional methods without adjustable parameters: The PBE0 model, J. Chem. Phys. 110, 6158 (1999).

[63] V. Tran, R. Soklaski, Y. Liang, and L. Yang, Layer-controlled band gap and anisotropic excitons in few-layer black phosphorus, Phys. Rev. B 89, 235319 (2014).

[64] J. Kim, S. S. Baik, S. H. Ryu, Y. Sohn, S. Park, B.-G. Park, J. Denlinger, Y. Yi, H. J. Choi, K. S. Kim, Observation of tunable band gap and anisotropic Dirac semimetal state in black phosphorus, Science 349, 723 (2015).

[65] J. Qiao, X. Kong, Z. X. Hu, F. Yang, W. Ji, High-mobility transport anisotropy and linear dichroism in few-layer black phosphorus, Nat. Commun. 5, 4475 (2014).

[66] H. J. Conley, B. Wang, J. I. Ziegler, R. F. Haglund, Jr. Sokrates, T. Pantelides, K. I. Bolotin, Bandgap Engineering of Strained Monolayer and Bilayer MoS2, Nano Lett. 13, 3626 (2013).

[67] K. F. Mak, C. Lee, J. Hone, J. Shan, T. F. Heinz, Atomically Thin $\mathrm{MoS}_{2}$ : A New Direct-Gap Semiconductor, Phys. Rev. Lett. 105, 136805 (2010).

[68] G. B. Liu, D. Xiao, Y. Yao, X. Xu, W. Yao, Electronic structures and theoretical modeling of two-dimensional group-VIB transition metal dichalcogenides, Chem. Soc. Rev. 44, 2643 (2015).

[69] J. Yang, R. Xu, J. Pei, Y. W. Myint, F. Wang, Z. Wang, S. Zhang, Z. Yu, and Y. Lu, Optical tuning of exciton and trion emissions in monolayer phosphorene, Light: Science and Applications 4, e312 (2015)

[70] O. Gritsenko, R. van Leeuwen, E. van Lenthe, and E. J. Baerends, Self-consistent approximation to the Kohn-Sham exchange potential, Phys. Rev. A 51, 1944 (1995).

[71] M. Kuisma, J. Ojanen, J. Enkovaara, and T. T. Rantala, KohnSham potential with discontinuity for band gap materials, Phys. Rev. B 82, 115106 (2010). 